\title{
Therapeutically Effective Controlled Release Formulation of Pirfenidone from Nontoxic Biocompatible Carboxymethyl Pullulan- Poly(vinyl alcohol) Interpenetrating Polymer Networks
}

\author{
Saundray Raj Soni, ${ }^{\dagger}$ Bibhas K. Bhunia, ${ }^{\ddagger}$ Nimmy Kumari, ${ }^{\dagger}$ Subhashis Dan, ${ }^{\prime}$ Sudipta Mukherjee, ${ }^{\S}$
}

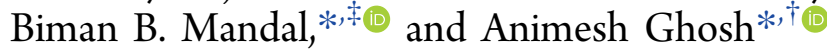

\begin{abstract}
${ }^{\dagger}$ Department of Pharmaceutical Sciences and Technology, Birla Institute of Technology, Mesra, Ranchi 835215, Jharkhand, India
${ }^{\ddagger}$ Biomaterial and Tissue Engineering Laboratory, Department of Biosciences and Bioengineering, Indian Institute of Technology Guwahati, Guwahati 781039, Assam, India

${ }^{\S}$ Division of Pharmaceutics, Department of Pharmaceutical Technology and "Bioequivalence Study Centre, Department of Pharmaceutical Technology, Jadavpur University, Kolkata 700032, India
\end{abstract}

Supporting Information

\begin{abstract}
The present study was conducted to develop therapeutically effective controlled release formulation of pirfenidone (PFD) and explore the possibility to reduce the total administered dose and dosing regimen. For this purpose, $\mathrm{pH}$-sensitive biomaterial was prepared by inducing carboxymethyl group on pullulan by Williamson ether synthesis reaction, and further, interpenetrating polymeric network microspheres were prepared by glutaraldehyde-assisted water-in-oil (w/o) emulsion cross-linking method, which showed higher swelling ratio in acidic and basic $\mathrm{pH}$. The formation of microspheres was confirmed by different spectral characterization techniques, and thermal kinetic study indicated the formation of thermally stable microspheres.

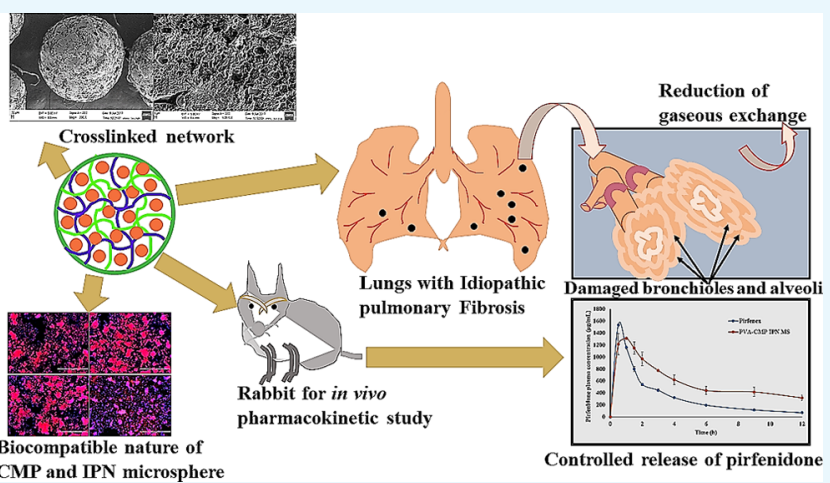
Cell viability and biocompatibility studies on hepatocellular carcinoma (HepG2) cell showed the polymeric matrix to be biocompatible. In vitro dissolution of optimized formulation (F5) showed releases of 54.09 and $76.37 \%$ in $0.1 \mathrm{~N} \mathrm{HCl}$ after $2 \mathrm{~h}$ and phosphate buffer ( $\mathrm{pH} 6.8)$ up to 8 h, respectively. In vivo performances of prepared microsphere and marketed product of PFD were compared in rabbit. $T_{\max }$ (time taken to reach peak plasma concentration) was found to be achieved at $0.83 \mathrm{~h}$, compared to $0.5 \mathrm{~h}$ for Pirfenex with no significant difference complementing the immediate action, while area under curve was significantly greater for optimized formulation ( $9768 \pm 1300$ $\mathrm{ng} \mathrm{h} / \mathrm{mL})$ compared to Pirfenex $(4311 \pm 110 \mathrm{ng} \mathrm{h} / \mathrm{mL})$, complementing the sustained action. In vivo pharmacokinetic study suggested that the prepared microsphere could be a potential candidate for therapeutically effective controlled delivery of PFD used in dyspnea and cough management due to idiopathic pulmonary fibrosis.
\end{abstract}

\section{INTRODUCTION}

Drug delivery through oral route is the most common delivery route for administration of drugs to elicit the therapeutic response. ${ }^{1}$ As per the reports of future market insights, the oral controlled release drug delivery technology global market will register a growth rate of $7.2 \%$ during 2017-2027 and the market will generate a revenue of U.S. $\$ 50000$ million by the end of 2027. An ideal dose in the treatment is the one that quickly attains the minimum therapeutic concentration in blood plasma and the concentration is maintained in the therapeutic window for the entire duration of treatment. The conventional sustained-release drug-delivery system predominantly maintains the plasma drug concentration for a longer period of time by deferring "time to reach peak plasma concentration" $\left(t_{\max }\right)$ in contrast to immediate-release drugdelivery system. So, minimum effective plasma concentration could be achieved quickly from immediate-release drugdelivery system, but at the same time, drug will not be available in gastrointestinal tract (GIT) for sustained absorption, as there is no release-controlling step in the dosage form, requiring a frequent dosing of the drug. ${ }^{3}$ The fluctuation of drug plasma concentration may lead to shoot beyond toxic level or remain below minimum therapeutic level, causing toxic manifestation in both the cases. ${ }^{4,5}$ So, there is always a need to develop a drug-delivery system that could be able to burst release the drug as to achieve $t_{\max }$ quickly for immediate action and thereafter release the drug in a precontrolled manner to maintain the plasma drug concen-

Received: May 2, 2018

Accepted: September 12, 2018

Published: September 26, 2018 
Table 1. Different Batches of Carboxymethyl Pullulan, Their Conditions of Preparation, Degree of Substitution Values, and Elemental Analysis ${ }^{a}$

\begin{tabular}{|c|c|c|c|c|c|c|c|}
\hline \multirow[b]{2}{*}{ batch code } & \multicolumn{3}{|c|}{ process variables } & \multirow[b]{2}{*}{ DS } & \multicolumn{3}{|c|}{ elemental analysis } \\
\hline & volume of $10 \mathrm{M} \mathrm{NaOH}(\mathrm{mL})$ & MCA (g) & temp $\left({ }^{\circ} \mathrm{C}\right)$ & & $\mathrm{C}(\%)$ & $\mathrm{H}(\%)$ & $\mathrm{O}(\%)$ \\
\hline NP & & & & & 41.06 & 7.72 & 51.22 \\
\hline CMP-1 & 5 & 5 & 65 & $0.296 \pm 0.056$ & 36.79 & 6.75 & 56.46 \\
\hline CMP-2 & 10 & 5 & 65 & $0.539 \pm 0.032$ & 30.91 & 5.29 & 63.80 \\
\hline CMP-3 & 15 & 5 & 65 & $0.395 \pm 0.029$ & 35.28 & 5.82 & 58.90 \\
\hline CMP-4 & 20 & 5 & 65 & $0.328 \pm 0.028$ & 35.22 & 6.46 & 58.32 \\
\hline CMP-5 & 5 & 2.5 & 65 & $0.157 \pm 0.025$ & 38.22 & 7.69 & 54.09 \\
\hline CMP-6 & 10 & 2.5 & 65 & $0.345 \pm 0.028$ & 35.64 & 6.35 & 58.01 \\
\hline CMP-7 & 10 & 5 & 95 & $0.433 \pm 0.030$ & 34.97 & 6.62 & 58.41 \\
\hline CMP-8 & 10 & 5 & 35 & $0.282 \pm 0.027$ & 36.91 & 7.29 & 55.80 \\
\hline CMP-9 & 2.5 & 2.5 & 65 & $0.116 \pm 0.024$ & 39.11 & 7.44 & 53.45 \\
\hline CMP-10 & 7.5 & 2.5 & 65 & $0.250 \pm 0.027$ & 36.05 & 7.29 & 56.66 \\
\hline CMP-11 & 12.5 & 2.5 & 65 & $0.314 \pm 0.000$ & 36.07 & 6.56 & 57.37 \\
\hline CMP-12 & 15 & 2.5 & 65 & $0.298 \pm 0.027$ & 36.29 & 7.01 & 56.70 \\
\hline CMP-13 & 10 & 7.5 & 65 & $0.505 \pm 0.031$ & 33.29 & 7.02 & 60.69 \\
\hline
\end{tabular}

tration for a longer duration of time. ${ }^{3}$ In the last few decades, application of natural polymer in controlled drug-delivery system has gained a significant importance in medical and biomedical applications. $^{6,7}$ The various different polymers harnessed include xanthan gum ${ }^{7}$ chitosan, ${ }^{8}$ gellan gum, ${ }^{9}$ locust bean gum, ${ }^{10}$ guar gum, ${ }^{11}$ etc. In most reports, it was observed that the in vitro release of $\operatorname{drug}(\mathrm{s})$ from the polymeric matrix sustained more than $24 \mathrm{~h}^{12-13}$ But the residence time of any solid oral formulation in gastrointestinal tract is as maximum as $8-10 \mathrm{~h}$ depending on the fed and fasted conditions unless the formulation is modified for specific targets. Therefore, a large amount of drugs still remains in the formulation matrix after $8-10 \mathrm{~h}$, which could not be available for absorption, meaning underutilization of delivered dose. ${ }^{4}$ Hence, there is a need to control the drug release from a rate-controlling polymer matrix within certain hours $(8-10 \mathrm{~h})$ to achieve maximum absorption of drug. The amount of rate-controlling polymer used in formulation is not only the factor that controls the release rate, but other factors such as swelling properties, network crosslinking sites, and density plays a crucial role to achieve preprogrammable release rate within a certain time periods. $^{16-19}$ Accordingly, there is a need to tailor the native polymer to incorporate the required properties.

Interpenetrating polymeric network (IPN) is one such innovative form of drug-delivery system, which comprises two or more networks, partially interlaced on a molecular scale, which cannot be separated until the chemical bonds are broken. ${ }^{20}$ Hence, there are many variables available in the IPN system, which ultimately gives us the opportunity to achieve intended specific requirements. Apart from modification of the polymer used as the backbone in IPN system, other variables such as the amount of cross-linker and, more specifically, drug polymer ratio will also be available to control the release rate within the transit time period. ${ }^{21}$

Idiopathic pulmonary fibrosis (IPF) is a chronic, catastrophic, and progressive lung disease with very-difficult-totreat situation, leading to respiratory failure and death within 3-5 years of diagnosis. The initial symptoms of IPF are dyspnea, cough, fatigue, depression, anxiety, relationship problem, and financial difficulties, of which dyspnea and cough are the serious issues for which management is immediately required. There is no evidence of cough suppression by opioids in IPF patients, while pirfenidone (PFD) has been proved to be effective with beneficial effects on a subgroup population suffering with IPF. ${ }^{22}$

Our aim is to develop a therapeutically effective controlled drug-delivery system for the symptomatic management of IPF, which will be able to release the required amount of drug as loading dose and rest of the drug in equivalent rate as maintenance dose. As PFD is used in management of dyspnea and cough in IPF patients, the time taken to achieve peak plasma concentration $\left(t_{\max }\right)$ should be as low as possible to get immediate relief from the dyspnea and cough. In a study conducted by the sponsors for approval filing to U.S. Food and Drug Administration, it was found that $45.80 \pm 8.24 \%$ of the ${ }^{14} \mathrm{C}$ PFD dose was absorbed from the stomach within 30 min. ${ }^{23}$ Hence, the swelling behavior of polymeric matrix must be $\mathrm{pH}$-dependent so that the release of drug from the matrix in stomach (acidic $\mathrm{pH}$ ) would be sufficient to meet the criteria of loading dose. Polymeric matrix must also be able to release the rest of the drug in intestine as to suffice the maintenance dose within the transit time of approximately $6 \mathrm{~h}$. The swelling characteristic of IPN microspheres using carboxymethylated polymer and poly(vinyl alcohol) (PVA) was studied by different scientists, which confirmed $\mathrm{pH}$-dependent swelling behavior, indicating a higher swelling index in alkaline medium. ${ }^{19,24,25}$ So, to develop IPN microsphere of PFD with similar characteristics, the native biopolymer pullulan was modified to carboxymethyl pullulan (CMP), to render it $\mathrm{pH}-$ sensitive before preparing IPN microsphere. The $\mathrm{pH}$ sensitivity is a prerequisite, as swelling followed by drug release will be affected by $\mathrm{pH}$ of the media.

Microsphere was prepared using glutaraldehyde (GA)assisted water-in-oil (w/o) emulsion cross-linking technique. Spectral characterization was performed to confirm the crosslinking and formation of IPN microsphere. Field emission scanning electron microscopy (FESEM) was performed to study the shape and surface morphology of microspheres. In vitro enzymatic degradation was performed to assure the degradability of microspheres. Biocompatibility study and cell viability study were conducted on human hepatocellular carcinoma (HepG2) cell lines using 3-(4,5-dimethylthiazol-2yl)-2,5-diphenyltetrazolium bromide (MTT) assay and cell imaging techniques. In vitro release profile and release kinetics 
Table 2. Weight Average Molecular Weight, Viscosity, Contact Angle, and Surface Energy Measurement of Native Pullulan and Carboxymethyl Pullulan ${ }^{a}$

$\begin{array}{lcccc}\text { batch code } & M_{\mathrm{w}}(\mathrm{g} / \mathrm{mol}) & \text { PDI } & \text { viscosity }(\mathrm{cP}) & \text { contact angle }(\mathrm{deg}) \\ \text { NP } & 8.51 \times 10^{5} & 1.922 & 98.50 & 73.04 \pm 0.75 \\ \text { CMP-1 } & 8.10 \times 10^{6} & 1.288 & 64.80 & 43.41 \pm 0.12 \\ \text { CMP-2 } & 4.47 \times 10^{6} & 1.163 & 60.20 & 33.7 \pm 6.6 \\ \text { CMP-3 } & 6.94 \times 10^{6} & 1.144 & 62.10 & 57.47 \pm 0.00 \\ \text { CMP-4 } & 1.30 \times 10^{7} & 1.006 & 61.40 & 62.6 \pm 3.3 \\ \text { CMP-5 } & 1.11 \times 10^{6} & 1.277 & 69.40 & 58.5 \pm 1.3 \\ \text { CMP-6 } & 9.14 \times 10^{5} & 1.126 & 68.10 & 63.99 \pm 0.21 \\ \text { CMP-7 } & 1.72 \times 10^{6} & 1.719 & 60.50 & 55.5 \pm 3.3 \\ \text { CMP-8 } & 4.30 \times 10^{6} & 1.002 & 62.90 & 56.3 \pm 1.2 \\ \text { CMP-9 } & 1.17 \times 10^{7} & 1.318 & 65.70 & 63.6 \pm 1.8 \\ \text { CMP-10 } & 1.39 \times 10^{6} & 1.219 & 66.40 & 71.2 \pm 1.8 \\ \text { CMP-11 } & 1.47 \times 10^{5} & 1.718 & 62.50 & 62.7 \pm 2.1 \\ \text { CMP-12 } & 1.26 \times 10^{6} & 1.316 & 63.10 & 50.4 \pm 1.7 \\ \text { CMP-13 } & 5.10 \times 10^{7} & 1.193 & 61.10 & 49.5 \pm 1.5 \\ \text { weight-average molecular weight; PDI: polydispersity index; cP: centipoise; deg: degree; mN/m: millinewtons per meter. }\end{array}$

${ }^{a} M_{\mathrm{w}}$ : weight-average molecular weight; PDI: polydispersity index; cP: centipoise; deg: degree; $\mathrm{mN} / \mathrm{m}$ : millinewtons per meter.

of all formulations (F1-F9) were explored. Finally, optimized formulation and marketed product (Pirfenex) were subjected to an in vivo pharmacokinetic (PK) study in rabbits and different PK parameters were compared, showing that the microsphere was able to control release for $12 \mathrm{~h}$, with $C_{\max }$ achieved in $0.83 \mathrm{~h}$, satisfying our goal. In this way, the developed formulation could be a suitable candidate for therapeutically effective controlled release formulation of PFD.

\section{RESULTS AND DISCUSSION}

2.1. Synthesis of Carboxymethyl Pullulan and Effect of Various Process Variables on Degree of Substitution (DS). Pullulan is composed of a linear chain of maltotriose units linked by $\alpha$ - $(1 \rightarrow 6)$ glycosidic bond and possess various hydroxyl groups in its structure, which are desirable for substitution of carboxymethyl groups. The reaction pathway for the synthesis of CMP is illustrated in Figure S1, Supporting Information.

All of the process variables were taken into consideration to maximize the DS as per Table 1.

2.1.1. Effect of Process Variables on DS. Degree of substitution is the initial and main characterization parameter for a successful carboxymethylation reaction. DS value was found to be highly dependent on the concentration of sodium hydroxide, MCA, and reaction temperature, as can be observed from Table 1 and Figure S2, Supporting Information. With increase in the concentration of sodium hydroxide up to 10 $\mathrm{mL}$, the DS value increased ( $\mathrm{DS}=0.539$ ) and then started to decrease with further increase of $\mathrm{NaOH}$ concentration (15 and $20 \mathrm{~mL}, \mathrm{DS}=0.395$ and 0.328 , respectively). The initial increase in DS value was due to the activation of polymer for the formation of alkoxide and facilitated the swelling of pullulan for enhanced diffusion and penetration of etherifying agent (MCA). The DS value was reduced on further increase of $\mathrm{NaOH}$ concentration due to the competition between the main reaction and the side reaction of $\mathrm{NaOH}$ and MCA, with the side reaction becoming predominant with increased production of glycolate. $^{26}$ The effect of MCA concentration was also studied, and at optimized concentration of $\mathrm{NaOH}$, the DS value initially increased with increasing MCA concentration and then reduced on further increase in MCA concentration (Figure S2b, Supporting Information), due to increased formation of glycolate with increased MCA. ${ }^{27}$ The effect of temperature on DS value was also studied, which showed the highest DS value $(0.539)$ at $65{ }^{\circ} \mathrm{C}$ and reduced values at $35{ }^{\circ} \mathrm{C}(0.282)$ and $95{ }^{\circ} \mathrm{C}(0.433)$ (Figure S2c, Supporting Information). The reduced DS value at reduced temperature was observed due to less swelling of polymer and lack of activation energy to initiate and drive the reaction, while increased temperature caused chain degradation and intramolecular elimination of water, leading to a reduced number of hydroxyl groups available for substitution. ${ }^{26}$ Thus, considering the above observation, reaction condition was optimized based on the highest DS value.

2.2. Formulation of PVA-CMP IPN Microspheres. In the present work, IPN microspheres were prepared using the (w/o) emulsion cross-linking technique. The synthetic biopolymer PVA was blended with CMP and interlaced on the backbone of CMP with the help of GA, a bifunctional chemical cross-linker, which formed acetal bond between the hydroxyl group of PVA and CMP. This resulted in the formation of interpenetrating polymer network, a threedimensional (3D) structure of hydrogels, which can be distinguished from the regular polymeric blends. ${ }^{28}$ The prepared microspheres were insoluble in water and showed controlled hydration property with drug entrapped in the polymeric network. The reaction pathway for the formation of IPN microsphere is presented in Figure S3, Supporting Information.

2.3. Physicochemical Characterization. 2.3.1. Elemental Analysis. The presence of different elements $(\mathrm{C}, \mathrm{H}$, and $\mathrm{O})$ in native pullulan and CMP was determined, and the results are presented in Table 1 . The results showed that there was a significant increment in oxygen content in all of the batches of CMP compared to native pullulan and highest for CMP-2 (63.80\%). This observation was due to the attachment of $-\mathrm{CH}_{2} \mathrm{COONa}$ group at the replaceable hydroxyl group of pullulan. The carbon and hydrogen contents were found to be reduced for CMP ( $\mathrm{C}=30.91 \%, \mathrm{H}=5.29 \%$, for CMP-2) compared to native pullulan ( $\mathrm{C}=41.06 \%, \mathrm{H}=7.72 \%)$ due to polymer chain distortion because of high concentration of $\mathrm{NaOH}$, vigorous stirring during the reaction, and participation of hydrogen in the formation of water molecules.

2.3.2. Viscosity Study. The viscosity of native pullulan was found to be $98.50 \mathrm{cP}$, which is higher compared to all CMP batches $(69.40-60.20 \mathrm{cP})$, as evident in Table 2. The reduced 
viscosity was observed due to the use of concentrated $\mathrm{NaOH}$ and monochloroacetic acid at elevated temperature for modification. This condition initiated breaking of polymeric backbone by $\beta$-elimination reaction at the reducing sugar units containing hydroxyl groups. Further, during the reaction in alkaline media, saccharinic acids are generated, which increases the aqueous solubility and reduces the viscosity. ${ }^{29}$

2.3.3. Weight Average Molecular Weight and Polydispersity Index. The effect of modification of native pullulan was clearly understood from the DS value, and molecular weight analysis revealed increased molecular weight of all batches of CMP compared to that of native pullulan $\left(8.51 \times 10^{5} \mathrm{Da}\right)$. The increment in molecular weight was due to the introduction of carboxymethyl group on the polymeric backbone, which affects the hydrodynamic volume of the polymer molecules and affects chain aggregation. Furthermore, pullulan is a nonionic polymer, while after introduction of the carboxymethyl group, it becomes polyelectrolyte (charged polymer molecule) and hence the molecular weights of ionic and nonionic biopolymers cannot be directly compared. The polydispersity index (PDI) of pullulan was 1.922, and for CMP, it was in the range of 1.719-1.002 (Table 2), indicating more uniform molecular mass distribution of CMP compared to pullulan. ${ }^{30}$

2.3.4. Contact Angle and Surface Energy Measurement. The study was conducted to visualize the changes in the wettability of CMP compared to native pullulan, and the results are shown in Table 2 and the images of contact angle are shown in Figure S4, Supporting Information. After carboxymethylation, the contact angle was found to be decreased with increased surface energy compared to native pullulan. The contact angle was found to be lowest for the highest value of DS and gradually increased with decreasing DS value, as evident from Tables 1 and 2 . The reason for this observation is the presence of highly polar $-\mathrm{COO}^{-}$group in CMP, which increases the affinity toward polar molecules like water and easily breaks its cohesive forces, resulting in increased wettability with reduced contact angle.

2.3.5. Fourier Transform Infrared (FTIR) Spectroscopy. FTIR spectroscopy was performed initially for pullulan and CMP to confirm the carboxymethylation of pullulan. The FTIR spectra of pullulan showed characteristic peaks at 3605 and $2918 \mathrm{~cm}^{-1}$ due to stretching vibrations of free hydroxyl $(-\mathrm{OH})$ and $-\mathrm{CH}_{2}$ groups, respectively. Further, various peaks were observed in the range of $1250-1070 \mathrm{~cm}^{-1}$ due to stretching vibrations of $\mathrm{C}-\mathrm{O}-\mathrm{H}$ and $\mathrm{C}-\mathrm{O}-\mathrm{C}$ from ether linkage. In case of FTIR spectra of CMP, a broad peak at 3622 $\mathrm{cm}^{-1}$ was observed, indicating the utilization of hydroxyl group in the chemical reaction. The characteristic peaks of CMP were observed due to $\mathrm{C}=\mathrm{O}$ stretching of $\mathrm{COO}^{-}$ions, $\mathrm{C}-\mathrm{O}$ stretching of ether, $\mathrm{C}-\mathrm{O}-\mathrm{H}$ stretching/bending, and $\mathrm{C}-\mathrm{O}-\mathrm{C}$ (symmetric) stretching at 1635, 1518, 1450, 1024, and 898 $\mathrm{cm}^{-1}$, respectively. ${ }^{31}$

The FTIR spectra of PVA (Figure 1a) showed the characteristic peak at $3622 \mathrm{~cm}^{-1}$ because of the presence of stretching vibration of free hydroxyl group. A sharp peak was observed at $2900 \mathrm{~cm}^{-1}$ due to $\mathrm{C}-\mathrm{H}$ vibration of alkyl group and at $1500 \mathrm{~cm}^{-1}$ due to the bending vibration of the $-\mathrm{CH}_{2}$ group present in PVA.

The FTIR spectra of PFD (Figure 1d) showed characteristic peaks at 3050,1531 , and $1668 \mathrm{~cm}^{-1}$ due to aromatic $\mathrm{C}-\mathrm{H}$ stretching vibration, aromatic $\mathrm{C}=\mathrm{C}$ stretching vibration, and stretching vibrations of tertiary amide group.

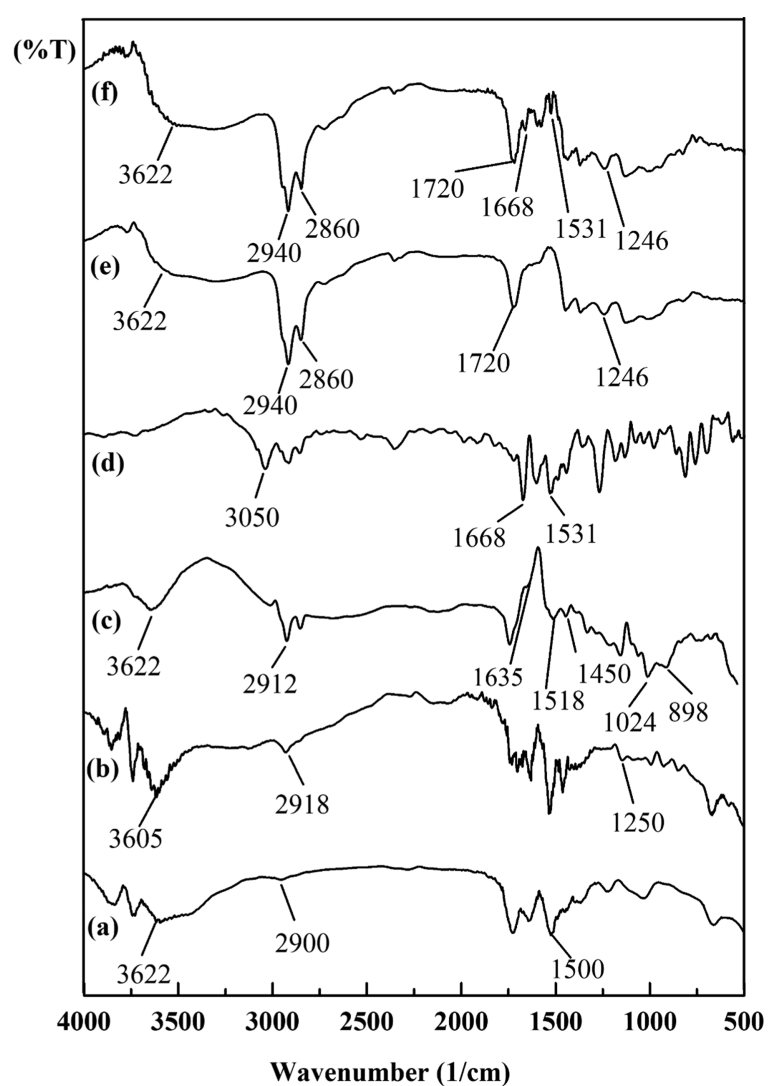

Figure 1. FTIR spectra of (a) PVA, (b) pullulan, (c) CMP, (d) pure drug, (e) placebo IPN, and (f) drug-loaded IPN.

The FTIR spectra of placebo microsphere (Figure 1e) showed broadened peak of hydroxyl group, indicating participation of hydroxyl group in acetal bond formation. Further, characteristic peaks at 2860 and $2940 \mathrm{~cm}^{-1}$ were observed due to aldehyde-related $\mathrm{C}-\mathrm{H}$ stretching, a duplet absorption with peaks contributed through alkyl chain. ${ }^{32} \mathrm{~A}$ peak at $1724 \mathrm{~cm}^{-1}$ was observed, which may be due to the $\mathrm{C}=$ $\mathrm{O}$ group of PVA acetate. The small peak at $1246 \mathrm{~cm}^{-1}$ and other broader absorption band in the range of 1050-1140 $\mathrm{cm}^{-1}$ was observed for ether and acetal linkage. The appearance of these new peaks confirmed the formation of IPN microspheres. Similarly, FTIR spectroscopy was performed for finished product (Figure 1f), which showed additional peaks at 1618 and $1531 \mathrm{~cm}^{-1}$, indicating the presence of drug, along with all of the peaks observed in placebo microspheres. This confirmed the formation of PFDloaded IPN microspheres.

2.3.6. Raman Spectroscopy. Raman spectroscopy was performed for PVA, pullulan, CMP, and CMP-PVA IPN microsphere. In the Raman spectra of pullulan (Figure $2 \mathrm{~b}$ ), the characteristic bands at 2913,1471 , and $1352 \mathrm{~cm}^{-1}$ were due to $\mathrm{C}-\mathrm{H}$ stretching, $\mathrm{C}-\mathrm{H}$ bending, and $\mathrm{O}-\mathrm{H}$ bending vibrations, respectively. The different characteristic peaks observed at $1132 \mathrm{~cm}^{-1}$ (broad band), and 941 and $858 \mathrm{~cm}^{-1}$ (sharp peaks) were attributed to $\mathrm{C}-\mathrm{O}-\mathrm{C}$ (asymmetric) and $\mathrm{C}-\mathrm{O}-\mathrm{C}$ stretching, respectively. The Raman spectra (Figure 2c) of CMP also showed the characteristic peaks of pullulan with an additional peak at $1752 \mathrm{~cm}^{-1}$ due to $\mathrm{C}=\mathrm{O}$ stretching of acid present in the carboxymethyl group.

The monomeric unit of PVA $\left(-\mathrm{CH}_{2}-\mathrm{CHOH}-\right)$ is mainly responsible for the vibrational spectra of PVA (Figure 2a). The 


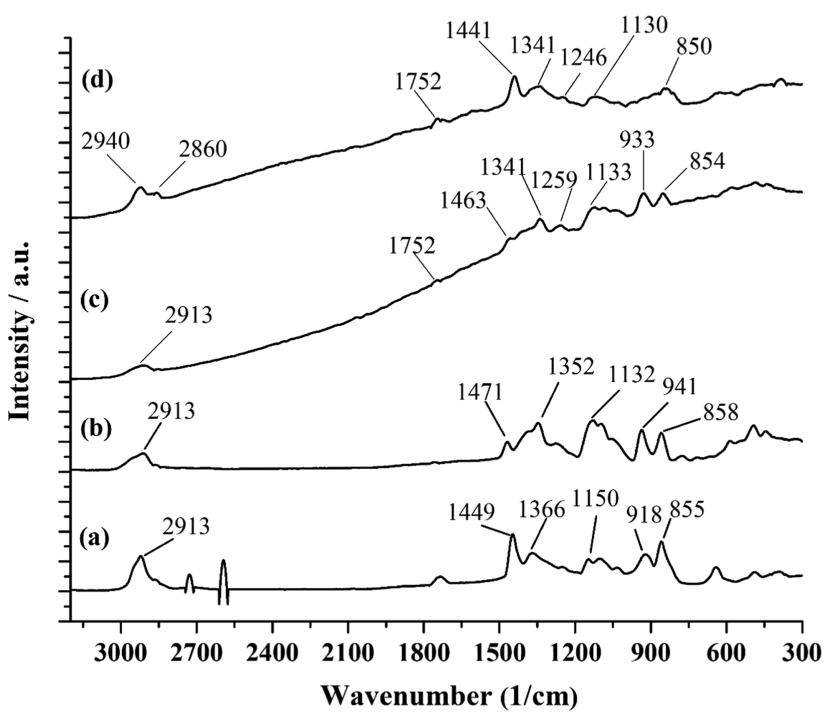

Figure 2. Raman spectra of (a) PVA, (b) pullulan, (c) CMP, and (d) placebo IPN.

prominent peaks at 2913,1449 , and $1366 \mathrm{~cm}^{-1}$ were due to $\mathrm{C}-\mathrm{H}$ stretching, $\mathrm{C}-\mathrm{H}$ bending, and $\mathrm{O}-\mathrm{H}$ bending vibrations, respectively. The $\mathrm{C}-\mathrm{O}$ stretching and $\mathrm{C}-\mathrm{C}$ stretching vibrations were observed at 1150 and $918 \mathrm{~cm}^{-1}$, respectively. These observed bands were at similar wavenumbers, as reported previously. ${ }^{33}$

In the case of IPN microsphere (Figure 2d), the duplet absorption peak was observed at 2940 and $2860 \mathrm{~cm}^{-1}$ due to aldehyde-related $\mathrm{C}-\mathrm{H}$ stretching contributed through alkyl chain. The peak at 1752 and $1441 \mathrm{~cm}^{-1}$ was observed due to $\mathrm{C}=\mathrm{O}$ stretching of acid and $\mathrm{C}-\mathrm{O}$ stretching of ether. The peaks observed at 1130,850 , and $804 \mathrm{~cm}^{-1}$ were due to acetal linkage $(\mathrm{C}-\mathrm{O}-\mathrm{C})$ formed during cross-linking reactions, confirming the formation of IPN microsphere.

2.3.7. Solid State ${ }^{13} \mathrm{C} N M R$ Spectroscopy. The NMR spectra of pullulan were presented in our previous work. ${ }^{21}$ The spectra showed distinct peaks for different carbon atoms, which was assigned unambiguously to all six carbon atoms. The peaks of C6 atom $(6 \mathrm{~g} 1 \rightarrow 4)$ and primary C6 atom $(4 \mathrm{~g}$ unit) were observed at $\delta=63.340$ and $62.314 \mathrm{ppm}$, respectively. The peak observed at $73.423 \mathrm{ppm}$ was broadened with increased intensity due to merging and overlapping of C2, C3, and C5 carbon atoms of pullulan. The peaks observed at 82.483 and 102.995 ppm were assigned to $\mathrm{C} 4$ and $\mathrm{C} 1$ carbon atoms of pullulan. Similarly, the peaks observed for CMP (Figure 3a) were also assigned for all of the carbon atoms. The spectra showed the appearance of additional peaks at 178.021 ppm compared to native pullulan, which indicated the presence of carbonyl group of carboxymethyl group at the 2$\mathrm{O}-$, 3-O-, and 6-O-positions. Further, the peak at $72.483 \mathrm{ppm}$ was observed due to the presence of methylene group at 2-O-, 3-O-, and 6-O-positions, which indicated successful modification of pullulan to carboxymethyl pullulan. The NMR spectra of PVA were reported earlier, ${ }^{34,35}$ which showed sharp peaks for $-\mathrm{CH}_{2}$ carbon and three split peaks due to the resonance carbon of PVA at 43 and $60-80$ ppm, respectively. The ${ }^{13} \mathrm{C}$ NMR spectra of placebo PVA-CMP IPN microsphere are shown in Figure 3b. The peak at $22.235 \mathrm{ppm}$ was assigned to the aliphatic carbon of the $-\mathrm{CH}_{2}$ group in PVA. Different peaks observed in the range of $37-42 \mathrm{ppm}$ (37.190, 39.327, and $44.112 \mathrm{ppm}$ ) were due to the aliphatic carbons of GA. The peak C6 carbon of CMP was observed at $68.809 \mathrm{ppm}$, and the peak at $73.936 \mathrm{ppm}$ (C2, C3, and C5 carbons of CMP) was broadened and observed with increased intensity due to merging of $\mathrm{CH}$ resonance peak of PVA and methylene group of carboxymethyl pullulan. The peak at $102.307 \mathrm{ppm}$ was due to the acetal carbon, confirming the formation of IPN microsphere. The last peak observed at $171.441 \mathrm{ppm}$ was due to the carbonyl carbon atom present in CMP. So, the NMR spectra confirmed carboxymethylation and the formation of GA-assisted cross-linked IPN microsphere.

2.3.8. X-ray Diffraction (XRD) Spectroscopy. The XRD spectra of pure drug showed intense peaks at $2 \theta$ values of 8.46 , $14.42,15.04,18.46,22.92,24.36,26.9$, and 32.4 , due to its crystalline nature. The XRD spectra of placebo IPN and drug loading (DL) IPN microspheres did not show any peak because of the amorphous nature of the polymer and the presence of drug in molecularly dispersed form in the polymer matrix (Figure S5, Supporting Information), respectively, and found to be consistent with previous reports. ${ }^{36,37}$

2.3.9. Differential Scanning Calorimetry (DSC). The DSC thermogram of PFD and PVA showed a sharp endothermic peak at 110.40 and $192.19{ }^{\circ} \mathrm{C}$, respectively, due to its melting transition. No peak was observed in the DSC thermogram of CMP. In the case of placebo and PFD-loaded IPN microsphere (Figure S6, Supporting Information), no sharp peak was observed; instead, a broad curve at $70{ }^{\circ} \mathrm{C}$ was observed due to the presence of moisture in the three-dimensional networks of microsphere. The absence of a sharp peak in the PFD-loaded microsphere indicated the presence of drug in the form of molecular dispersion in the polymer matrix and suggested a consistent result with that of XRD.

2.3.10. Field Emission Scanning Electron Microscopy. The surface morphologies of pullulan, CMP, and PVA-CMP IPN were investigated by FESEM (Figure 4). The FESEM images indicated that the native polymer was oval, ellipsoidal, and irregular shaped, while the modified pullulan was rough with sharp end break point geometry. The change in the geometry may be due to breaking of polymeric backbone during chemical reaction because of the application of highly concentrated alkali and MCA. The surface morphology and cross-sectional area of prepared microsphere were also investigated to confirm the network formation. Figure $4 \mathrm{c}$ shows a group of microsphere, and Figure $4 \mathrm{~d}$ shows the FESEM image of a single microsphere. From the image, it was observed that the prepared microsphere was spherical in shape and rough in morphology with numerous tiny pores present on the surface, indicating the formation of porous polymeric matrix, which may become a factor for diffusion-dominant release behavior of drug from microspheres. The FESEM image of the cross-sectional area of microsphere (Figure $4 \mathrm{e}$ ) revealed a highly cross-linked network structure responsible for swelling and confirmed the successful formation of IPN network inside microsphere.

2.4. Thermal Kinetic Study. 2.4.1. Thermogravimetric Analysis (TGA). Thermogravimetric analysis was performed for $\mathrm{CMP}$, the three different placebo microspheres prepared using different concentrations of GA $(1,3$, and $5 \mathrm{~mL})$, pure drug $\mathrm{PFD}$, and optimized formulation (F5) using thermogravimetric analyzer (TGA-4000, PerkinElmer). TGA and differential thermal gravimetric (DTG) (first derivative) curves were plotted using double $Y$ axis graph against temperature $(\mathrm{K})$ for each analyzed sample, as presented in Figures S7-S12, Supporting Information. The TGA curve of CMP showed a 

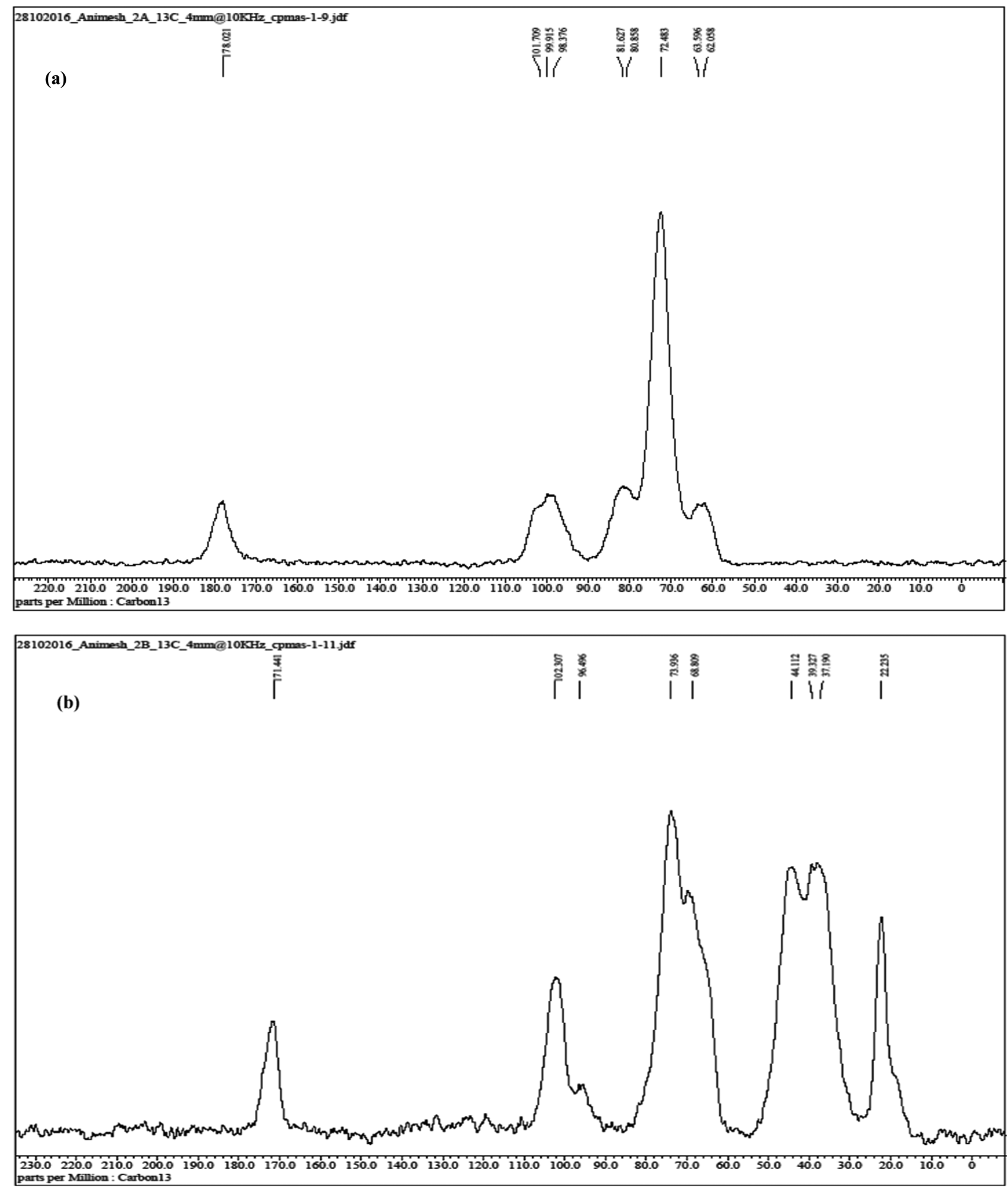

Figure $3 .{ }^{13} \mathrm{C}$ NMR spectra of (a) carboxymethyl pullulan and (b) placebo PVA-CMP IPN microsphere.

single-step major degradation of $67.32 \%$ in the temperature range of 507-630 K, supported by DTG curve showing the sharp peak of degradation at $552 \mathrm{~K}$ at a heating rate of $5 \mathrm{~K} /$ min. The degradation peak of CMP at different heating rates 5, 10,15 , and $20 \mathrm{~K}$ was found to be slightly shifted toward higher temperatures $552,564,567$, and $578 \mathrm{~K}$, respectively, as shown in the DTG curve of Figure S7, Supporting Information. The observation was due to heat transfer lag in the heating rate. ${ }^{38}$ Earlier, we had reported the thermal analysis of pullulan performed with similar methodology, which showed a singlestep sharp degradation peak at $590 \mathrm{~K}$. The observed difference suggested structural changes during carboxymethylation; furthermore, the presence of sodium ion of sodium carboxymethyl group in the CMP also alters the thermal 

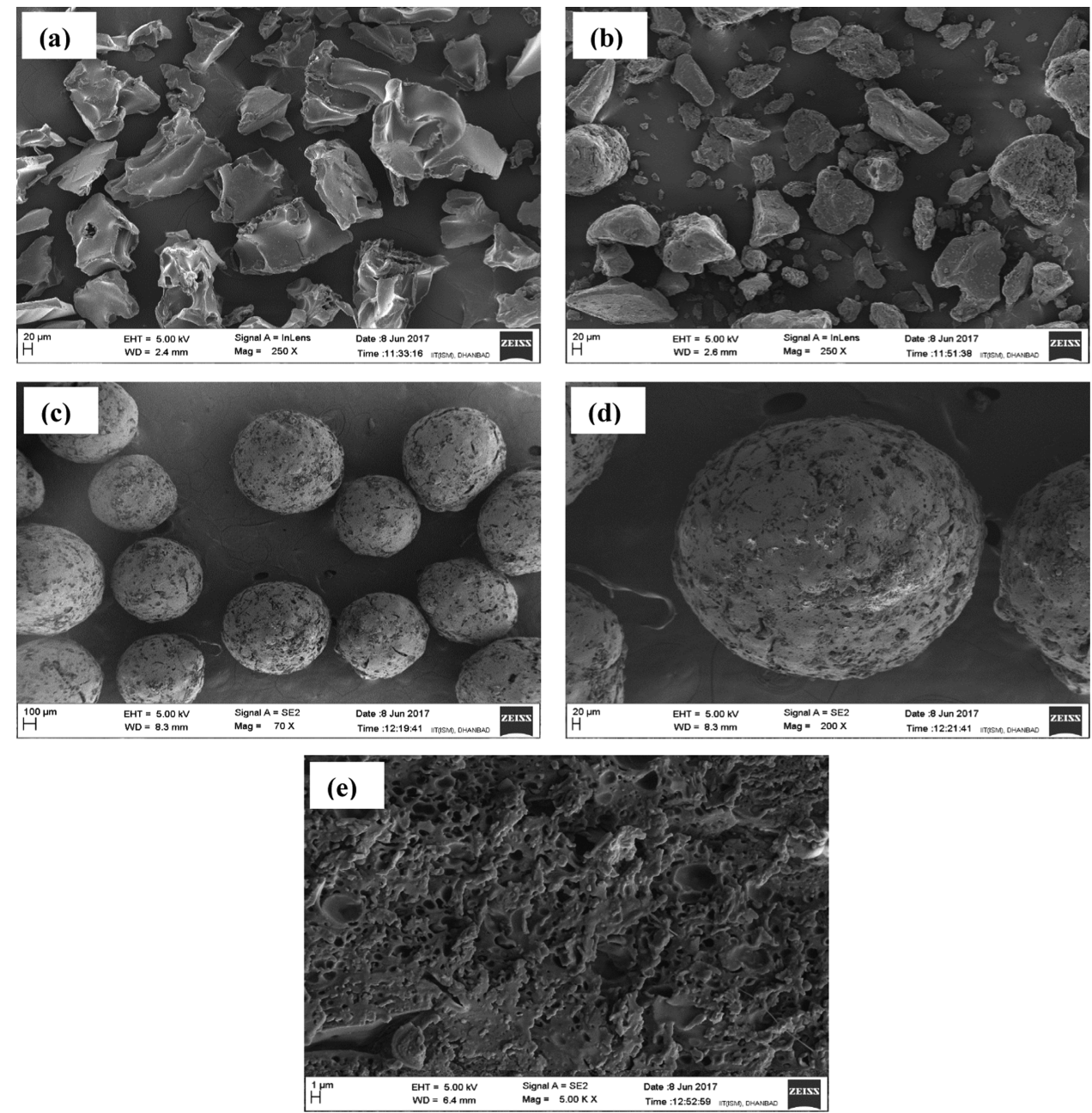

Figure 4. FESEM images of (a) pullulan, (b) carboxymethyl pullulan, (c) group of microspheres, (d) single microsphere, and (e) cross-linked network in the polymer matrix.

decomposition pattern, ${ }^{39}$ which confirmed successful modification of pullulan.

The TGA and DTG curves of PVA were reported in our previous study, ${ }^{21}$ which indicated a sharp degradation peak at $600 \mathrm{~K}$ due to rapid chain fastening and elimination of $\mathrm{H}_{2} \mathrm{O}$, converting the polyenes to aliphatic group by Diels-Alder intramolecular cyclization or radical reactions. Upon progress of reaction, the $\mathrm{C}-\mathrm{C}$ cleavage and its carbonization occurred at 720 and $750 \mathrm{~K}$, respectively. ${ }^{40}$

The TGA and differential thermal analysis (DTA) curves of IPN microsphere showed different degradation patterns from its individual components. In the case of microsphere, four degradation zones were observed in the range of 300-850 K. The first degradation was observed as a small hump in the range of $363-435 \mathrm{~K}$ with a nominal weight loss of $4.83 \%$, which could be due to loss of moisture entrapped in the polymeric network. The second zone of degradation was from 526 to $678 \mathrm{~K}$, with a weight loss of $55.47 \%$, indicating the major degradation zone. The DTG curve in this range showed a broad peak at $647 \mathrm{~K}$ for lower heating rates (5 and $10 \mathrm{~K}$ / $\mathrm{min})$, which became comparatively sharper for heating rates (15 and $20 \mathrm{~K} / \mathrm{min}$ ) due to increased heating rate. This indicated the formation of IPN microsphere as the degradation zone of CMP (507-630 K) and PVA (495-650 K) broadened and shifted in the range of 526-678 $\mathrm{K}$, which included rapid breakage of polymeric chain into smaller units with elimination of the hydroxyl group. The third step included breakage of carbon chains $(\mathrm{C}-\mathrm{C})$, as evident with the sharp degradation peak at $706 \mathrm{~K}(682-718 \mathrm{~K})$, and the fourth degradation zone $718-789 \mathrm{~K}$ indicated carbonization and formation of tars of carbon units.

The thermal analysis of pure drug PFD showed a single-step degradation at $510 \mathrm{~K}$ at a heating rate of $5 \mathrm{~K} / \mathrm{min}$ degradation range $(429.28-515 \mathrm{~K})$, which was shifted to higher temperature with increase in heating rate (Figure S11, Supporting Information).

Thermogram of optimized formulation (F5) showed a similar pattern to placebo microsphere (Figure S12, Supporting Information). The degradation zone of drug was clearly visible in the range of $427-544 \mathrm{~K}$, but the degradation pattern and zones remain the same, indicating the stability of the IPN matrix in the presence of drug.

This thermal observation supported the formation of IPN microsphere, and their TGA and DTG curves at different temperatures are shown in Figures S8-S10, Supporting Information.

2.4.2. Activation Energy. Activation energy $\left(E_{\mathrm{a}}\right)$ was calculated using the Ozawa-Flynn-Wall (OFW) and Kissinger-Akahira-Sunose (KAS) models. These models yielded a slope value (Figure S13, Supporting Information) from the 
linear relationship between $\ln (\beta)$ vs $1 / T$ (eq 2 ) and the slope (Figure S14, Supporting Information) of graph $\ln \left(\beta / T^{2}\right)$ vs $1 /$ $T$ when multiplied with gas constant $(R=8.314 \mathrm{~J} /(\mathrm{mol} \mathrm{K}))$. Slope activation energy as a function of degree of conversion $(\alpha)$ for all of the samples is graphically shown in Figure 5.
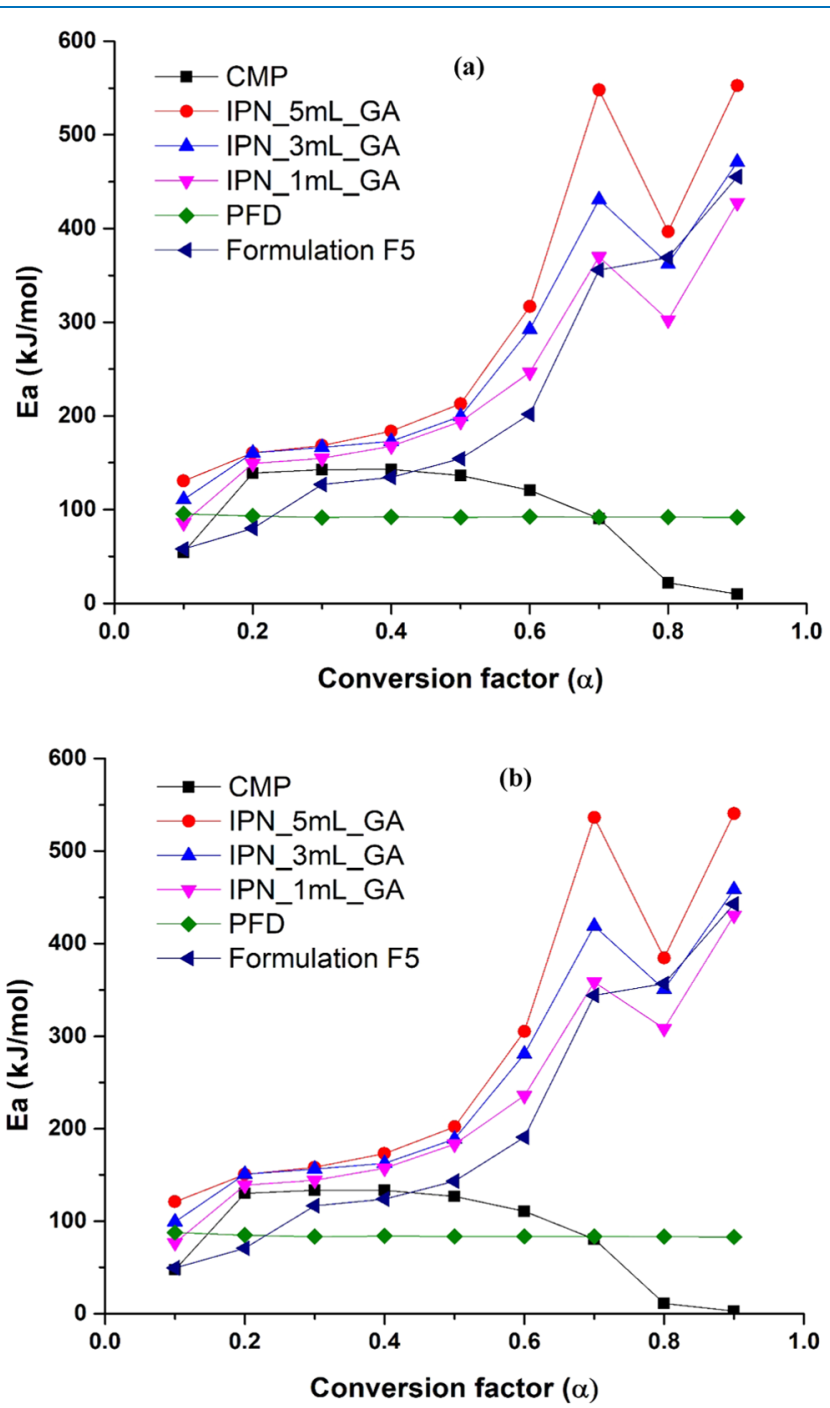

Figure 5. Activation energy as a function of the conversion factor $(\alpha)$ using (a) OFW model and (b) KAS model.

In our previous report, ${ }^{21}$ activation energy of pullulan was calculated, which showed initial higher activation energy due to high energy requirement to initiate the glycosidic bond cleavage, and once the polymeric chain started to decompose, the $E_{\mathrm{a}}$ value started to decrease. CMP showed an entirely different pattern of $E_{\mathrm{a}}$ value at different conversion factors due to harsh condition (use of concentrated $\mathrm{NaOH}$ and MCA) during carboxymethylation process, causing scissions of the helical coiled structure at the functional groups and the glycosidic linkage, resulting in restructuring and substitution of carboxymethyl group at the hydroxyl group. Initially, the activation energy for CMP was the minimum at 0.1 (54.46 and $47.33 \mathrm{~kJ} / \mathrm{mol}$ for OFW and KAS models, respectively) and the energy required for next $10 \%$ degradation $(\alpha=0.2)$ was almost 3 times (138.9 and $129.98 \mathrm{~kJ} / \mathrm{mol}$ for OFW and KAS models, respectively), which was constant up to $\alpha=0.6$, showing major degradation of polymeric chain in this region, and then significantly decreased, as evident from Figure 5.

IPNs showed an increasing trend in the activation energy with increasing conversion factor $(\alpha)$. The activation energy was minimum at $\alpha=0.1$ and increased up to $\alpha=0.7$, which showed that high energy was required with increasing step for polymeric chain cleavage due to the formation of cross-linked network structure (formation of IPN), and then decreased at $\alpha$ $=0.8$, indicating decomposition of almost polymeric chain and network. At this stage, different pyrolysis byproducts (dehydrated products of carbon structure) like alkane, alkene, dienes, and aromatic cyclization of carbonaceous char residues were formed and required high activation energy for its conversion to carbon tars as evident at $\alpha=0.9$, in Figure 5, and at this stage, weight loss was about $99 \%$ and no residue was left. ${ }^{41}$ This kind of observation was consistent with all of the three IPN microspheres and activation energy increased with increasing concentration of cross-linker at a fixed conversion factor.

The activation energy of the optimized formulation was found to be slightly reduced (Figure 5) in the entire range of $\alpha$ $=0.1-0.9$. The observation may be due to the effect of drug, so the $E_{\mathrm{a}}$ value of drug was also calculated, which on an average was found to be $92.49 \mathrm{~kJ} / \mathrm{mol}$, much lower than placebo IPNs. The increasing trend of $E_{\mathrm{a}}$ value with increasing conversion factor suggests the formation of stable PFD-entrapped IPN microsphere.

The data fitted in both the models showed almost parallel regression lines $\left(R^{2}>0.99\right)$ in the conversion range $(0.1<\alpha<$ 0.9 ), indicating the applicability of the OFW and KAS models for the calculation of activation energy. ${ }^{41}$ Upon analysis of the $E_{\mathrm{a}}$ values of both models, the value for the KAS model is lower than that of the OFW model, which can be due to the different approximations of the temperature integral. However, both the models show similar trend without any significant difference on $E_{\mathrm{a}}$ with conversion factor under consideration (0.1-0.9), indicating the chosen isoconversional models to be reasonably true.

2.5. In Vitro Enzymatic Degradation Study. In vitro action of pullulanase (E.C. No. 3.2.1.41) was studied on CMP and PVA-CMP IPN microsphere (placebo) to assure the biodegradation of synthesized novel products. Pullulanase is a debranching enzyme, which specifically hydrolyzes $\alpha-(1 \rightarrow 6)$ glycosidic bond. ${ }^{42}$ The experimentally obtained data were plotted as a digestibility curve of CMP (Figure 6a) and PVACMP IPN microsphere (Figure $6 \mathrm{~b}$ ). The digestibility curve of CMP showed faster digestion (45.77\% after $8 \mathrm{~h}$ ) compared to IPN microsphere $(41.97 \%$ after $48 \mathrm{~h})$. This indicated a very low digestion rate for IPN microsphere due to the formation of 3D polymer network during cross-linking reaction, compared to CMP, which was present in its two-dimensional structure and the $\alpha-(1 \rightarrow 6)$ glycosidic bond was easily accessible by the enzyme.

The log of slope (LOS) plot for the degradation data was made for CMP and IPN microsphere, which showed two degradation phases for both the test materials (Figure $6 c, d$ ). The LOS plot yielded two straight lines after it was fitted in the first-order kinetic equation (eq 11). Degradation rate constant $(k)$ in $\min ^{-1}$ was calculated from the slope value, and $C_{\infty}$ form the intercept of the straight line equation generated from the first-order kinetic equation ${ }^{43,44}$ (Table S1, Supporting Information). The digestibility rate constant for CMP was higher than IPN microsphere in both the phases due to the 
(a)

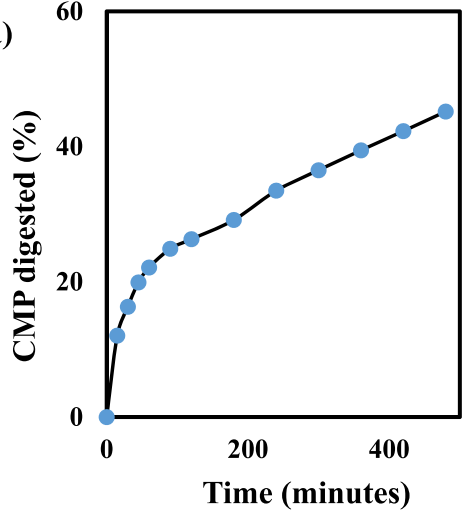

(c)

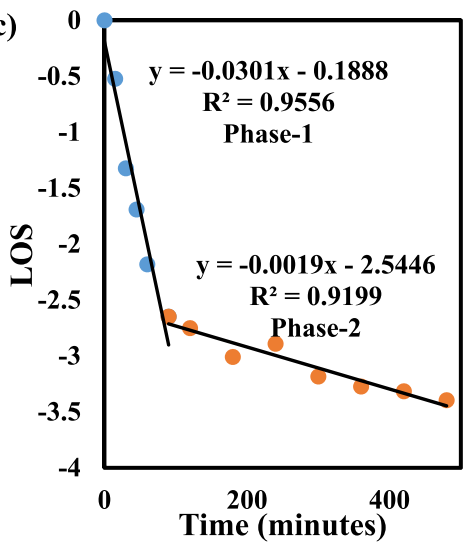

(b)

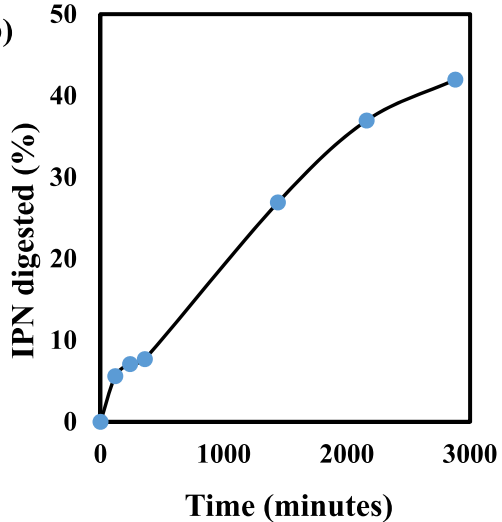

(d)

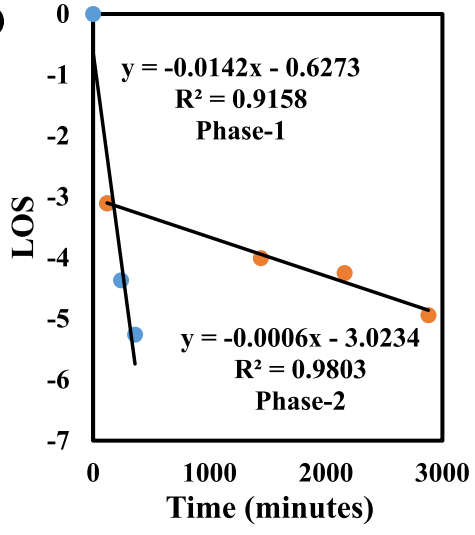

Figure 6. Digestibility curves of (a) CMP and (b) CMP-PVA IPN microsphere, and logarithm of slope (LOS) plots of (c) CMP and (d) CMPPVA IPN microsphere.

(a)

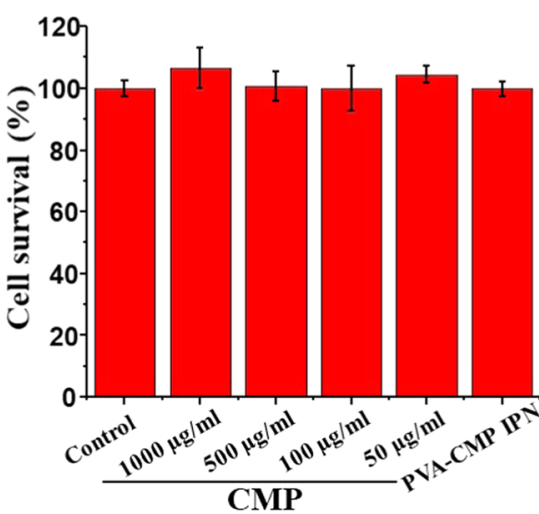

(b)
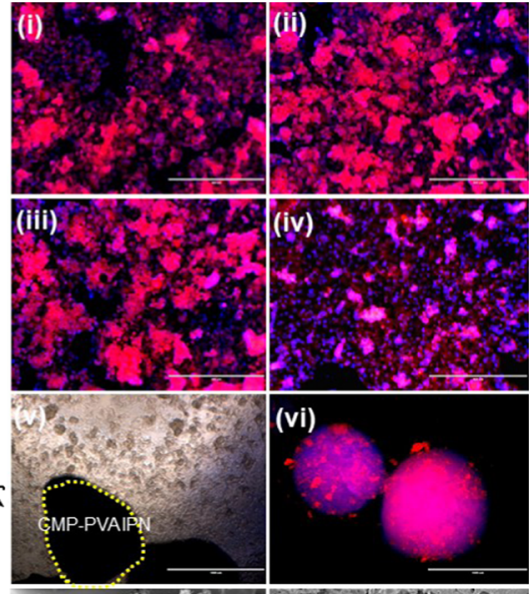

(vi)

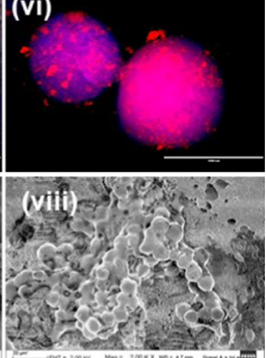

Figure 7. Viability of the human hepatocellular carcinoma (HepG2) cells detected by MTT assay at $72 \mathrm{~h}$. (a) Dose-dependent cell proliferation assay of CMP and direct contact of PVA-CMP IPN microsphere. (b) Rhodamine-phalloidin (stains actin)/Hoechst (stains nucleolus) staining of cells treated at different concentrations: (i) $1000 \mu \mathrm{g} / \mathrm{mL}$, (ii) $500 \mu \mathrm{g} / \mathrm{mL}$, (iii) $50 \mu \mathrm{g} / \mathrm{mL}$ of CMP, (iv) control, (v) phase contrast image of cells adhered to microsphere, (vi) rhodamine-phalloidin/Hoechst staining of adhered cells on microsphere, and (vii, viii) FESEM images of cell adhered microsphere at $270 \times$ and $2000 \times$, respectively. (Data are presented as mean $\pm \operatorname{SD}(n=3)$.) The scale bar for the images (i) $-($ iv $)$ is $400 \mu \mathrm{m}$ and for (v) $-(\mathrm{vi})$ is $1000 \mu \mathrm{m}$.

linear structure of CMP in which the $\alpha$ - $(1 \rightarrow 6)$ glycosidic bond was easily accessible compared to sterically hindered target sites of microsphere due to network formation. This is also evident from the values of final concentration $\left(C_{\infty}\right)$, as reported in Table S1, Supporting Information. Furthermore, the LOS graph yielded a linear regression line $\left(R^{2}>0.91\right)$, 
Table 3. Formulation Variables, \% Yield, Particle Size $d$ (0.5), Polydispersity Index, \% Drug Entrapment Efficiency, and Equilibrium Water Uptake in $\mathrm{pH} 1.2$ and $6.8^{a}$

\begin{tabular}{|c|c|c|c|c|c|c|c|c|}
\hline \multirow[b]{2}{*}{ formulation code } & \multirow[b]{2}{*}{$\mathrm{GA}(\mathrm{mL})$} & \multirow[b]{2}{*}{$\%$ drug loading } & \multirow[b]{2}{*}{ yield (\%) } & \multirow[b]{2}{*}{ particle size $[d(0.5)](\mu \mathrm{m})$} & \multirow[b]{2}{*}{ PDI } & \multirow[b]{2}{*}{$\operatorname{DEE}(\%)( \pm \mathrm{SD}, n=3)$} & \multicolumn{2}{|c|}{$\begin{array}{l}\text { equilibrium water } \\
\text { uptake }\end{array}$} \\
\hline & & & & & & & pH 1.2 & $\mathrm{pH} 6.8$ \\
\hline $\mathrm{F} 1$ & 3 & 45 & 66.76 & 956.42 & 0.905 & $39.62 \pm 0.69$ & 156.41 & 212.09 \\
\hline $\mathrm{F} 2$ & 1 & 30 & 62.77 & 972.26 & 0.910 & $25.84 \pm 0.50$ & 193.89 & 273.57 \\
\hline F3 & 3 & 30 & 74.54 & 939.12 & 0.947 & $29.4 \pm 1.9$ & 170.15 & 219.72 \\
\hline $\mathrm{F} 4$ & 5 & 30 & 83.81 & 822.47 & 0.884 & $36.43 \pm 0.95$ & 158.33 & 205.38 \\
\hline F5 & 5 & 45 & 72.24 & 835.16 & 0.950 & $46.72 \pm 0.49$ & 139.75 & 188.89 \\
\hline F6 & 3 & 15 & 86.78 & 926.87 & 0.880 & $27.82 \pm 0.62$ & 183.72 & 223.51 \\
\hline F7 & 1 & 45 & 54.90 & 989.15 & 0.877 & $32.9 \pm 2.1$ & 180.53 & 261.47 \\
\hline F8 & 5 & 15 & 92.96 & 810.12 & 0.896 & $31.45 \pm 0.73$ & 161.02 & 209.35 \\
\hline F9 & 1 & 15 & 75.30 & 958.09 & 0.909 & $22.56 \pm 0.19$ & 202.38 & 305.55 \\
\hline
\end{tabular}

confirming the first-order digestibility model to be a suitable predictor for enzymatic degradation in our test materials.

2.6. In Vitro Cytotoxicity Test and Biocompatibility Assessment. The modified biopolymer will be used for the formulation dosage forms for oral route, so it is essential to confirm the noncytotoxic and biocompatible nature of the material. The MTT assay for CMP and PVA-CMP IPN microsphere was performed at four different concentrations $(1000,500,100$, and $50 \mu \mathrm{g} / \mathrm{mL})$ and with direct contact to microsphere and compared to control. After $72 \mathrm{~h}$ of incubation, the results showed no significant difference in the cell viability (data presented as mean \pm standard deviation (SD, $n=3)$ ), as shown in Figure 7 a. Biocompatibility assessment was performed by cell imaging technique, which used fluorescent dye rhodamine-phalloidin for actin staining and Hoechst 33342 for nucleus staining. These images did not show any remarkable change in cellular morphology compared to control (Figure $7 \mathrm{~b}$-i-iv). FESEM image analysis showed that cells were adhered and spread onto the surface of microsphere (Figure $7 \mathrm{~b}$-vii,viii), indicating the noncytotoxic and biocompatible nature of the biopolymer and its microsphere.

2.7. Acute Oral Toxicity Study. The hematological and serum biochemical analysis reports are presented in Tables S2 and S3, Supporting Information. These results indicated normal levels of hematological and serum biochemical parameters, and no significant deviation was observed in test animals compared to control animals. The histopathological images of the vital organs like heart, liver, kidney, and stomach did not showed any significant difference (Figure S15, Supporting Information). Further zero mortality of the animals were observed, indicating the $\mathrm{LD}_{50}$ value of CMP and PVACMP IPN microsphere to be greater than the recommended dose $(2000 \mathrm{mg} / \mathrm{kg})$ of body weight. Therefore, CMP and PVA-CMP IPN microsphere were classified under "category 5" with "zero toxicity" indicating safe for drug-delivery application through oral route.

2.8. Characterization of PFD-Loaded PVA-CMP IPN Microsphere. 2.8.1. Estimation of Percentage Yield. The total amount of microsphere prepared for each formulation (F1-F9) was calculated in terms of percentage yield, and is shown in Table 3. The yield was found to be dependent on the concentration of GA and percentage drug loading (\% DL) in the system. The results showed that yield was found to be decreased with increasing drug loading due to limited solubility of PFD $(20 \mathrm{mg} / \mathrm{mL})$ and remained unentrapped in the system.
The second factor (GA concentration) showed increased yield of final product, with increased GA concentration. The result was in good agreement as the increased concentration of GA enhanced breaking of polymeric chain and exposed more hydroxyl group available for cross-linking and formation for acetal bond between PVA and CMP in the emulsion system. The mathematical relationship generated (in coded factors) for percentage yield was as follows

$$
\begin{aligned}
y_{1} & =75.02+9.34 x_{1}-10.19 x_{2}-0.080 x_{1} x_{2}-2.20 x_{1}^{2} \\
& +1.28 x_{2}^{2} \quad\left(R^{2}=0.9950\right)
\end{aligned}
$$

The predicted $R^{2}$ of 0.9502 is in agreement with the adjusted $R^{2}$ of $0.9887(p \leq 0.05)$.

where $y_{1}$ is the percentage yield of final product, $x_{1}$ and $x_{2}$ are the quantity of GA $(\mathrm{mL})$ and percentage $\mathrm{DL}$, respectively, and its contour plot is shown in Figure S16a, Supporting Information.

2.8.2. Drug Entrapment Efficiency (DEE). The percentage DEE of the prepared microspheres (F1-F9) was calculated and found in the range of $22.56 \pm 0.19$ to $46.72 \pm 0.49 \%$, presented in Table 3. The entrapment efficiency of the microsphere was found to be increasing with increasing concentration of GA at similar \% DL (at 15\% DL, F9 < F6 $<\mathrm{F} 8$; at 30\% DL, F2 < F3 < F4, and at 45\% DL, F7 < F1 < F5) values shown in Table 3 . The observation was due to the reason that increased concentration of GA increased the crosslinking density, producing highly cross-linked polymer networks, thus preventing the leaching of drug from polymer matrices. The increased drug content in the emulsion system also increased DEE at the similar concentration of GA, due to more availability of PFD in the system for cross-linking. ${ }^{45}$ The mathematical relationship generated (in coded factors) for drug entrapment efficiency is as follows

$$
\begin{aligned}
y_{2} & =29.95+5.56 x_{1}+6.23 x_{2}+1.24 x_{1} x_{2}+0.58 x_{1}^{2} \\
& +3.17 x_{2}^{2} \quad\left(R^{2}=0.9932\right)
\end{aligned}
$$

The predicted $R^{2}$ of 0.9496 is in agreement with the adjusted $R^{2}$ of $0.9848(p \leq 0.05)$.

where $y_{2}$ is the drug entrapment efficiency, $x_{1}$ and $x_{2}$ are the quantity of GA (mL) and percentage $\mathrm{DL}$, respectively, and its contour plot is shown in Figure S16b, Supporting Information.

2.8.3. Particle Size Analysis. The arithmetic mean diameter [d (0.5)] was found, and also the polydispersity index of microspheres (F1-F9) was found in the range of 810.12- 
989.15 $\mu \mathrm{m}$ (Table 3). The formulations with high concentration of GA with same \% DL showed smaller particle size due to increased cross-linking density and more squeezing of internal phase, resulting in decreased void space. The increased particle size of microspheres with higher \% DL was observed due to the presence of high concentration of drug in the emulsion system to be incorporated in the polymeric matrices. The polydispersity index of the formulations was found to be in the range of $0.877-0.950$, indicating a narrow size distribution range, supporting formation of microspheres prepared with uniform particle size. The histogram and particle size distribution of the optimized formulation are shown in Figure S17, Supporting Information.

2.8.4. Swelling Study. Swelling study of prepared microspheres (F1-F9) was conducted in terms of its equilibrium swelling ratio (\% ESR) at two different $\mathrm{pH}$ buffers ( $\mathrm{pH} 1.2$ and 6.8). Higher swelling ratio was observed in microspheres prepared by carboxymethyl pullulan in contrast to microspheres prepared by native pullulan. ${ }^{21}$ Higher swelling ratio (Table 3) in acidic media was due to enhanced hydrophilicity induced by highly polar carboxymethyl group in carboxymethyl pullulan. ${ }^{25}$ The carboxymethyl group ionizes when $\mathrm{pH}$ of the media is above the $\mathrm{p} K_{\mathrm{a}}$ value $(3.4-3.7)$ of carboxylic group. Higher swelling ratio (Table 3 ) in basic media was due to the availability of free carboxylic groups to form $\mathrm{H}$-bond with water. ${ }^{46}$ The swelling behavior was also dependent on the concentration of GA; results showed decreased swelling ratio for increased GA concentration, which was due to the increased extent of cross-linking and the consequent formation of rigid polymeric matrix, ${ }^{47}$ which prohibited the penetration of water into polymeric network. Percentage drug loading showed a slight increase in \% ESR with reduced drug loading, probably due to reduced internal space on polymeric matrix with increased drug quantity. A similar kind of swelling behavior was observed at both the $\mathrm{pH}(1.2$ and 6.8$)$ for IPN microspheres prepared using carboxymethylated polymer and PVA in different reports. ${ }^{19,24}$ This swelling property was considered to be instrumental in achieving low value of $t_{\max }$ required to get immediate relief from dyspnea and cough in IPF and thereafter to release the required drug within the transit time of intestine in sustained fashion as maintenance dose.

2.8.5. In Vitro Drug Release and Its Kinetic Modeling. 2.8.5.1. In Vitro Drug Release. In vitro drug release was performed for all in-house formulation (F1-F9) to explore the drug-release profile, which showed release profile to be dependent on the concentration of GA and drug loading ratio. The cumulative percentage drug release versus time (h) is graphically presented in Figure 8.

The drug release profile was found to be dependent on GA concentration. The microspheres showed decreased drug release with increased GA concentration $(1,3$, and $5 \mathrm{~mL})$ at constant $\%$ DL. ${ }^{48,49}$ For instance at 1,3 , and $5 \mathrm{~mL}$ of GA at constant DL value (15\%), the percentage cumulative drug release decreased and was found to be 90.98, 49.38, and $34.82 \%$ after $2 \mathrm{~h}$ (in $0.1 \mathrm{~N} \mathrm{HCl}$ ) and 99.82, 85.04, and 63.83\% after $8 \mathrm{~h}$ (in $\mathrm{PB} \mathrm{pH}$ 6.8) for formulations F9, F6, and F8, respectively. The effect of drug loading at a fixed amount of $\mathrm{GA}(1 \mathrm{~mL})$ did not show any sustained action because $1 \mathrm{~mL}$ of GA could not form rigid network structure required to release the drug in a sustained fashion (F9 (15\% DL), F2 (30\% DL), and F7 (45\% DL)), while drug loading has the effect on drug release at a higher amount of GA (5 mL) (F8 (15\% DL), F4

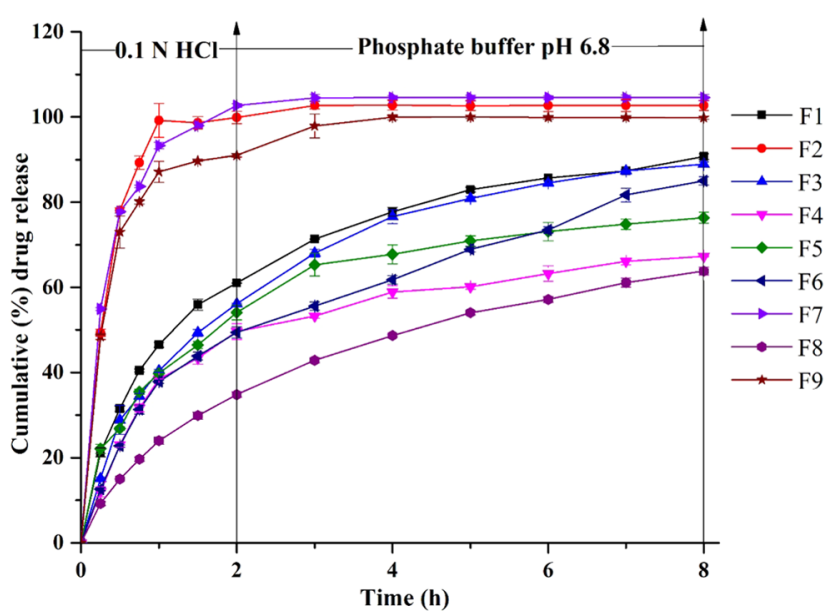

Figure 8. Release profile of PFD from different formulations (F1-F9) in $0.1 \mathrm{~N} \mathrm{HCl}$ and phosphate buffer $(\mathrm{PB}) \mathrm{pH} 6.8$.

(30\% DL), and F5 (30\% DL)). As the drug release mechanism is governed by the diffusion process, ${ }^{50}$ the increased release drug pattern with increase in \% DL was due to high drug concentration in the polymeric matrix, which in turn increased the concentration gradient and fastened the diffusion process by reducing polymeric barrier. At the same time, formulations with less \% DL faced increased hindrance to cross the matrix barrier, which reduced the diffusion process and overall controlled the drug release for an increased time period.

2.8.5.2. Kinetic Modeling of Drug Release. Zeroth-order ${ }^{51}$ and first-order kinetic models ${ }^{52}$ (equation details provided in Section S.4.10.1, Supporting Information) were applied to evaluate the drug-release kinetics for all of the batches of microspheres (F1-F9). In the case of first-order kinetic model, the $R^{2}$ value for all of the batches (Table S5, Supporting Information) was found to be greater than that of the zeroorder kinetic model, confirming first-order drug-release kinetics, i.e., microspheres release the drug in such a programmed manner that it is proportional to the amount of drug remaining in the matrix in such a way that the amount of drug released by unit of time diminish. ${ }^{53}$ In 1995, Muyle and Turko reported that pharmaceutical dosage forms containing water-soluble drugs in porous matrices follow first-order kinetics and our finding is consistent with the reports of Muyle and Turko, ${ }^{54}$ as PFD is a highly water-soluble drug. The mechanism of drug release was studied using the HixsonCrowell model, ${ }^{55}$ Higuchi model, ${ }^{56}$ and Korsmeyer-Peppas model, ${ }^{57}$ whose equations are provided in Section S.4.10.1, Supporting Information. The $n$ value of formulations F2, F7, and F9 was found to be less than 0.45 , which indicated that these formulations followed Fickian diffusion release mechanism. The $n$ value for all other formulation was higher than 0.45 (Table S5, Supporting Information), indicating nonFickian diffusion process. The $R^{2}$ value of the Higuchi model was found to be greater than the Hixson-Crowell model, indicating the diffusion process to be predominant over erosion of polymer matrix for the release of PFD from microspheres.

2.8.5.3. Statistical Analysis of the Formulation Variables on Release Rates: Drug Release after $2 \mathrm{~h}$ in $0.1 \mathrm{~N} \mathrm{HCl}$ and Drug Release after $8 \mathrm{~h}$ in Phosphate Buffer $\mathrm{pH}$ 6.8. The mathematical relationship generated using Design-Expert software (trial version 7.0.0, Stat-Ease, Inc., Minneapolis) between variable and responses (in coded factors) is as follows 


$$
\begin{aligned}
y_{3} & =57.04-25.84 x_{1}+7.10 x_{2}+1.89 x_{1} x_{2}+16.81 x_{1}^{2} \\
& -2.75 x_{2}^{2} \quad\left(R^{2}=0.9976\right)
\end{aligned}
$$

The predicted $R^{2}$ of 0.9780 is in agreement with the adjusted $R^{2}$ of $0.9945(p \leq 0.05)$.

$$
\begin{aligned}
y_{4} & =88.35-16.61 x_{1}+3.82 x_{2}+1.96 x_{1} x_{2}-2.69 x_{1}^{2} \\
& +0.17 x_{2}^{2} \quad\left(R^{2}=0.9946\right)
\end{aligned}
$$

The predicted $R^{2}$ of 0.9474 is in agreement with the adjusted $R^{2}$ of $0.9879(p \leq 0.05)$.

where $y_{3}$ is the percentage drug release after $2 \mathrm{~h}$ in $0.1 \mathrm{~N}$ $\mathrm{HCl}, y_{4}$ is the percentage drug release after $8 \mathrm{~h}$ in phosphate buffer $\mathrm{pH}$ 6.8, and $x_{1}$ and $x_{2}$ are the GA quantity and percentage DL, respectively, and its contour plot is shown in Figure S16c,d, Supporting Information.

Loading dose and maintenance dose were calculated based on eqs 5 and 6 , and it was found that maintenance dose should not be less than $26.58 \%$ of the total dose. Therefore, final optimized formulation was chosen based on the highest entrapment and "release after $2 \mathrm{~h}$ " not less than $26.58 \%$. Hence, F5 was chosen as optimized formulation with the highest entrapment $(46.72 \% \pm 0.49)$ and $54.09 \%$ drug release after $2 \mathrm{~h}$.

$$
\begin{aligned}
& D_{\mathrm{i}}=\frac{C_{\mathrm{ss}}}{F} \times V_{\mathrm{d}} \\
& D_{\mathrm{m}}=\frac{C_{\mathrm{L}}}{F} \times C_{\mathrm{ss}} \times T
\end{aligned}
$$

where $D_{i}$ is the loading dose, $C_{s s}$ is the steady-state concentration, $V_{\mathrm{d}}$ is the volume of distribution, $T$ is the time period, and $F$ is the bioavailability.

2.9. Stability Study. Stability study was conducted on the optimized formulation (F5) to study the effect of environmental conditions on time. The drug stability results (description, assay value, extent of drug release in 2 and $8 \mathrm{~h}$ (Table S6, Supporting Information) and complete dissolution profile (Figure S18, Supporting Information)) showed consistent performance. The assay value after 6 months was found to be $97.1 \pm 2.1 \%$ (standard assay value $95-105 \%$ as per PFD manufacturer certificate of analysis), which was within limit values, confirming that the developed formulation (F5) was stable for 6 months at accelerated conditions.

2.10. In Vivo Pharmacokinetic Study of PFD in Rabbits. Marketed tablet of PFD (Pirfenex) and in-house formulation (F5), equivalent to $15 \mathrm{mg} / \mathrm{kg}$ body weight were administered to rabbits of groups I and II, respectively. The plasma drug concentration was measured at predetermined time intervals, graphically presented in Figure 9. Different pharmacokinetic parameters like $C_{\max }, t_{\max }, \mathrm{AUC}_{0-t}, \mathrm{AUC}_{0-\infty}$, $t_{1 / 2}, K_{\mathrm{el}}$, and area under the first moment curve (AUMC) and mean residence time (MRT) values of both the formulations were calculated and compared in Table 4.

Time to achieve peak plasma concentration $\left(t_{\max }\right)$ predicts the rate of absorption and $t_{\max }$ value of F5 $(0.83 \pm 0.17 \mathrm{~h})$, and Pirfenex $(0.5 \pm 0.00 \mathrm{~h})$ did not show any significant difference $(p<0.05)$ indicating that the rate of absorption for formulation F5 was similar to that for Pirfenex. Therefore, immediate relief from dyspnea and cough in idiopathic pulmonary fibrosis could be achieved within approximately 1 $\mathrm{h}$ of dosing. Area under curve (AUC) measures the extent of

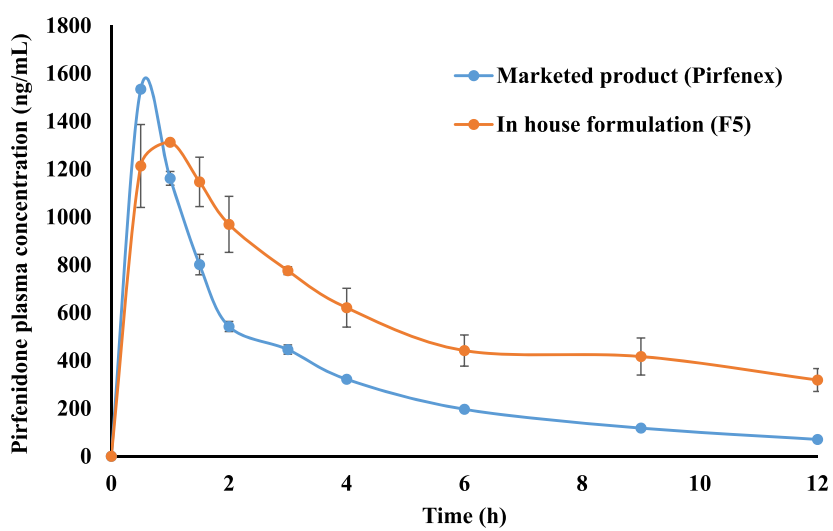

Figure 9. In vivo performances of marketed product (Pirfenex) and in-house formulation (F5) in rabbit plasma. Data presented as avg. \pm $\operatorname{SEM}(n=3)$.

absorption, which was significantly greater for formulation F5 (Table 4), indicating prolonged duration of action. This suggested that the matrix of IPN microsphere could efficiently control the in vivo drug release and maintain the drug plasma level in therapeutic window up to $12 \mathrm{~h}$. Finally, this formulation could be a promising delivery system in terms of immediate relief from dyspnea and cough along with reduction of dose as well as dosing frequency by maintaining the plasma drug concentration within the therapeutic window up to $12 \mathrm{~h}$.

\section{CONCLUSIONS}

In the present study, a $\mathrm{pH}$-sensitive therapeutically effective IPN microsphere was prepared. Spectral characterization like FTIR spectroscopy confirmed the drug polymer compatibility, and solid-state ${ }^{13} \mathrm{C}$ NMR spectra confirmed the formation of GA-assisted IPN microsphere. FESEM images revealed the spherical morphology of microspheres. Acute oral toxicity study confirmed the material to be nonhemotoxic in nature and classified in "category 5" with toxicity rating of "zero", confirming safe for use in drug-delivery applications. The CMP and IPN microsphere were found to be digested by enzyme pullulanase, confirming its biodegradable nature. The cell viability and biocompatibility experiments showed the polymeric material and formulation to be biocompatible as no significant decrease in cell percentage viability was observed even at $1000 \mu \mathrm{g} / \mathrm{mL}$ up to $72 \mathrm{~h}$. Swelling study of prepared microspheres showed $\mathrm{pH}$ sensitivity as comparatively higher swelling was observed in both acidic and alkaline media compared to swelling ratio of IPN microspheres prepared by native pullulan, which was essential to burst release the drug to get $t_{\max }$ as low as possible and further to control the release rate to achieve sustained action. In vivo pharmacokinetic parameters showed that $t_{\max }$ was found to be shifted to 0.83 $\mathrm{h}$, in contrast to $0.5 \mathrm{~h}$ for Pirfenex, the difference was not significant $(p<0.05)$, indicating that rate of absorption was similar to Pirfenex, while the AUC value for microsphere increased significantly and release was controlled up to $12 \mathrm{~h}$. Thus, in vivo pharmacokinetic study revealed successful formulation of therapeutically effective controlled release formulation of PFD.

\section{EXPERIMENTAL SECTION}

4.1. Materials. Pirfenidone (PFD) was received as a kind gifted sample from Biophore India Pharmaceuticals Pvt. Ltd., 
Hyderabad, Telangana, India. Pullulan was also obtained as a gifted sample from the principle manufacturer Hayashibara, Japan, supplied by Gangwal Chemicals, Mumbai, India. Poly(vinyl alcohol) (PVA) $88 \%$ hydrolyzed (from Thermo Fisher Scientific, Mumbai, India), light liquid paraffin (from HiMedia Laboratories Pvt. Ltd., Mumbai, India), Tween-80, hydrochloric acid $(\mathrm{HCl})$, glycine, petroleum ether (from Rankem, Gurgaon, Haryana), glutaraldehyde (GA) (from Loba Chemie, Mumbai, India), Dulbecco's modified Eagle's medium, fetal bovine serum, antibiotic solution containing (100 U/mL penicillin and $100 \mu \mathrm{g} / \mathrm{mL}$ streptomycin), amphotericin-B solution $(250 \mathrm{ng} / \mathrm{mL})$, trypsin-ethylenediaminetetraacetate (EDTA) solution (Gibco, Life Technologies), rhodamine-phalloidin (Invitrogen), Hoechst 33342, Triton-X100 , bovine serum albumin (BSA), and 3-(4,5-dimethylthiazol2-yl)-2,5-diphenyltetrazolium bromide (MTT) (from Sigma, St. Louis) were used as received. Milli-Q water was used throughout the experiment.

4.2. Synthesis of Sodium Carboxymethyl Pullulan (CMP). Carboxymethyl pullulan (CMP) was synthesized by the Williamson synthesis reaction from pullulan using the same method as reported earlier by our group with slight modification in reaction parameters and conditions. ${ }^{25}$ Detailed synthetic procedures are given in Section S.4.2, Supporting Information.

4.2.1. Determination of Degree of Substitution (DS) for Carboxymethyl Group on Pullulan. DS was calculated by the potentiometric back titration method using the equation below. ${ }^{58}$ The detailed methodology is discussed in Section S.4.2.1, Supporting Information.

$$
\mathrm{DS}=\frac{0.162 A}{(1-0.058 A)}
$$

where $A$ is the milliequivalents of $\mathrm{NaOH}$ required per gram of sample.

4.3. Preparation of Placebo PVA-CMP IPN Microspheres. The IPN microspheres were prepared using GAassisted water-in-oil (w/o) emulsion cross-linking method, as previously reported by our group ${ }^{25,59}$ with slight modification. Detailed synthetic procedures are given in Section S.4.3, Supporting Information.

4.4. Physicochemical Characterization. Elemental analysis, viscosity measurement, molecular weight analysis, contact angle, and surface energy measurement were performed for CMP, while Fourier transform infrared (FTIR) spectroscopy, Raman spectroscopy, solid-state ${ }^{13} \mathrm{C}$ NMR spectroscopy, X-ray diffraction (XRD) spectroscopy, differential scanning calorimetry (DSC), and field emission scanning electron microscopy (FESEM) analysis were performed for PVA, pullulan, CMP, PFD, and GA cross-linked PVA-pullulan IPN microspheres (placebo and drug loaded). These characterization studies were performed by established methods, and the detailed methodology is discussed in Section S.4.4, Supporting Information.

4.5. Thermal Kinetic Study. The thermal kinetic studies of CMP, different blank IPN microspheres (GA: 1, 3, and 5 $\mathrm{mL}$ ), pure drug PFD, and optimized formulation (F5) were performed with the same methodology as reported earlier. ${ }^{21}$ The study was performed using a thermogravimetric analyzer TGA-4000 (PerkinElmer). The samples were taken in a quantity of approximately 5-7 $\mathrm{mg}$ in a ceramic pan, placed in the furnace chamber, and heated at four different heating rates $(5,10,15$, and $20 \mathrm{~K} / \mathrm{min})$ from 300 to $900 \mathrm{~K}$ at constant flow 
of nitrogen gas at $20 \mathrm{~mL} / \mathrm{min}$. The activation energy $\left(E_{\mathrm{a}}\right)$ was calculated using two different isoconversional kinetic models, i.e., Ozawa-Flynn-Wall (OFW) $)^{60,61}$ and Kissinger-AkahiraSunose (KAS) $)^{62,63}$ for different conversion factors $(\alpha)$ from 0.1 to 0.9 , using eqs 8 and 9 , respectively, and the detailed discussion is provided in Section S.4.5, Supporting Information.

$$
\begin{aligned}
& \ln \left(\beta_{i}\right)=\left[\ln \left(\frac{A_{\alpha} R}{E_{\mathrm{a} i}}\right)-\ln f(\alpha)\right]-\left(\frac{E_{\mathrm{a} i}}{R T_{\alpha i}}\right) \\
& \ln \left(\frac{\beta_{i}}{T_{\alpha i}^{2}}\right)=\left[\ln \left(\frac{A_{\alpha} R}{E_{\alpha i}}\right)-\ln f(\alpha)\right]-\left(\frac{E_{\alpha i}}{R T_{\alpha i}}\right)
\end{aligned}
$$

4.6. In Vitro Enzymatic Degradation Study. In vitro degradation study was conducted using enzyme pullulanase (Sigma, St. Louis, E.C. No. 3.2.1.41). Both the test substances, CMP and microsphere, were incubated in a water bath at 37 ${ }^{\circ} \mathrm{C}$, and an aliquot of $1 \mathrm{~mL}$ of sample was withdrawn at predetermined time intervals. These samples were analyzed by reducing sugar assay method ${ }^{64,65}$ as described in Section S.4.6, Supporting Information.

4.6.1. Predictive Modeling for Digestibility. The degradation data were best fitted to first-order equation, and its firstorder derivative yielded logarithm of slope (LOS). ${ }^{43,44}$ Different parameters like digestibility rate constant $(k)$ and the concentration of the product at the end point of reaction $\left(C_{\infty}\right)$ were calculated from LOS plot using the following equations

$$
\begin{aligned}
& C_{t}=C_{\infty}\left(1-\mathrm{e}^{-k t}\right) \\
& \ln \left(\frac{\mathrm{d} C}{\mathrm{~d} t}\right)=-k t+\ln \left(C_{\infty} k\right)
\end{aligned}
$$

where $C_{t}$ is the product concentration at time $(t), C_{\infty}$ is the product concentration at the end of the reaction, $k$ is the digestibility rate constant, and $\ln \left(\frac{\mathrm{d} C}{\mathrm{~d} t}\right)$ is the logarithm of slope.

4.7. In Vitro Cytotoxicity Test and Biocompatibility Assessment. 4.7.1. Cell Viability Study. Cell viability study was performed using the 3-(4,5-dimethylthiazol-2-yl)-2,5diphenyltetrazolium bromide (MTT) (Sigma) assay, as described earlier. ${ }^{66}$ Cell culture and maintenance are discussed in Section S.4.7.1, Supporting Information. HepG2 cells (180 $\mu \mathrm{L}$ ) were seeded in a 96-well flat-bottomed plate at a concentration of $1 \times 10^{4}$ cells/well and incubated at $37{ }^{\circ} \mathrm{C}$ in a humidified atmosphere of $5 \% \mathrm{CO}_{2}$ for $24 \mathrm{~h}$. Thereafter, the cells were treated with $20 \mu \mathrm{L}$ of various concentrations $(1000-50 \mu \mathrm{g} / \mathrm{mL}$ ) of CMP (previously sterilized by UV light for three cycles $30 \mathrm{~min}$ each, and dissolved in incomplete medium) for $72 \mathrm{~h}$. For microspheres, $1 \times 10^{4}$ cells were seeded onto preconditioned samples leaving in undisturbed condition to allow cells adherence on them. Control wells did not contain any test sample except $20 \mu \mathrm{L}$ of phosphate-buffered saline (PBS). At predetermined time frame, spent media was replaced by fresh media $(180 \mu \mathrm{L})$ and aliquot of $20 \mu \mathrm{L}$ of MTT from stock $(5 \mathrm{mg} / \mathrm{mL})$ solution was added to each well. The plate was then incubated at $37{ }^{\circ} \mathrm{C}$ for $3 \mathrm{~h}$ and then replaced by $200 \mu \mathrm{L}$ of dimethyl sulfoxide to solubilize formazan crystal. Absorbance was taken at $570 \mathrm{~nm}$ using a multiplate reader (Tecan Infinite 200 PRO series, Switzerland). Cell viability was measured as percentage reduction of
MTT compared to control assigning $100 \%$ viability. Data were reported as mean \pm standard deviation $(\mathrm{SD})$ of three different independent observations.

4.7.2. In Vitro Biocompatibility Test. Biocompatibility of CMP and its microsphere was accessed by visualizing cellular morphology using cell imaging technique with fluorescent dyes and FESEM analysis.

4.7.2.1. Cell Imaging. To visualize cellular morphology, cells were stained with rhodamine-phalloidin fluorescent dye (Invitrogen) following the previously described protocol. ${ }^{67}$ Briefly, prior to staining, cells of 3 days culture were fixed in $10 \%$ formalin solution (Sigma) overnight at room temperature. The fixed cells were then washed in PBS and incubated with $1 \%$ bovine serum albumin (BSA, Sigma) for $30 \mathrm{~min}$ to inhibit the nonspecific binding of dye. This was further followed by PBS washing and permeabilization with $0.1 \%$ Triton-X-100 (Sigma) for $5 \mathrm{~min}$. After thorough PBS washing, the cells were stained with rhodamine-phalloidin $(0.165 \mu \mathrm{M})$ for $20 \mathrm{~min}$ in the dark. To visualize the nucleus, the cells were counterstained with $1 \mu \mathrm{g} / \mathrm{mL}$ Hoechst 33342 (Sigma) for $30 \mathrm{~min}$. The images were recorded using a fluorescent microscope (EVOS XL digital, Invitrogen).

4.7.2.2. Field Emission Scanning Electron Microscopy (FESEM) Study. FESEM study was performed for the cellseeded microspheres of 3 days culture. These cells were fixed in $2.5 \%$ GA for $3 \mathrm{~h}$ at room temperature, followed by gradual dehydration with ethanol $(30-100 \% \mathrm{v} / \mathrm{v})$ and subsequently allowed to dry completely in vacuum condition. The samples were then mounted on an aluminum stub, followed by gold sputter coating for $130 \mathrm{~s}$ before analysis. The images were recorded by a field emission electron microscope (Zeiss, Sigma, Germany) at an operating voltage of $2 \mathrm{kV}$ with a working distance of $5 \mathrm{~mm}$.

4.8. Acute Oral Toxicity Study. Acute oral toxicity studies of CMP and PVA-CMP placebo IPN microspheres were conducted as per Organization of Economic Co-operation and Development guideline for the test of chemicals 425, adopted on 17 December 2001. Details of the experimental procedure are provided in Section S.4.8, Supporting Information.

4.9. Preparation of PFD-Loaded PVA-CMP IPN Microspheres. The drug-loaded microspheres were prepared by adding required amount of PFD to the aqueous phase, and the remaining process was similar to that described in Section 4.3.

4.9.1. Formulation Variables and Factorial Design. In the present study, $3^{2}$ full factorial design was adopted and a total of nine formulations were prepared using the two factors [quantity $(\mathrm{mL})$ of cross-linking agent $\left(X_{1}\right)$ and $\%(\mathrm{w} / \mathrm{w})$ drug loading ratio $\left(X_{2}\right)$ ] at three different levels [high level $(+1)$, medium level $(0)$, and low level $(-1)]$. The percentage yield $\left(Y_{1}\right)$, drug entrapment efficiency $\left(Y_{2}\right)$, cumulative percentage drug release in $0.1 \mathrm{~N} \mathrm{HCl}$ after $2 \mathrm{~h}\left(Y_{3}\right)$ and in phosphate buffer after $8 \mathrm{~h}\left(Y_{4}\right)$ were selected as the responses. Mathematical equation model was generated by $3^{2}$ factorial design, ${ }^{68}$ using Design-Expert software (trial version 7.0.0, Stat-Ease, Inc., Minneapolis) to analyze the generated responses from dependent variables.

$$
y=b_{0}+b_{1} x_{1}+b_{2} x_{2}+b_{12} x_{1} x_{2}+b_{11} x_{1}^{2}+b_{22} x_{2}^{2}
$$

The different formulations according to the factorial design are presented in Table S4, Supporting Information, and the contour plot of responses is presented in Figure S16, Supporting Information. 
4.10. Characterization of PFD-Loaded PVA-CMP IPN Microspheres. Drug-loaded microspheres were characterized for their percentage yield, drug entrapment efficiency, particle size, and equilibrium swelling behavior as per the previously reported method. ${ }^{69}$

4.10.1. In Vitro Drug Release Study and Kinetic Modeling. In vitro drug release profile for all of the prepared formulations (F1-F9) were studied using USP type-1 dissolution apparatus (Electro Lab TDT-08L, Mumbai, India) at $100 \mathrm{rpm}$ and $37 \pm$ $0.5{ }^{\circ} \mathrm{C}$. The dissolution media used was $0.1 \mathrm{~N} \mathrm{HCl}$ for initial 2 $\mathrm{h}$ and further continued in phosphate buffer $\mathrm{pH} 6.8$ up to $8 \mathrm{~h}$. The samples were withdrawn at predetermined intervals, diluted suitably, and analyzed at $311 \mathrm{~nm}$ in a UV-visible spectrophotometer (UV-1800, Shimadzu, Japan). The average percentage cumulative drug release $\pm \operatorname{SD}(n=3)$ was calculated and plotted against each time interval. The drug release kinetics and mechanism were studied using different models, which are described in Section S.4.10.1, Supporting Information.

4.10.2. Stability Study. The optimized formulation (F5) was subjected to stability study loading as per International Conference on Harmonization guidelines (Q1E, step 4) for oral finished dosage form under accelerated conditions, ${ }^{70}$ and details are provided in Section S.4.10.2, Supporting Information.

4.11. In Vivo Pharmacokinetic Study. 4.11.1. Estimation of PFD in Rabbit Plasma. PFD content in rabbit plasma was estimated for the marketed formulation Pirfenex and the microsphere optimized formulation (F5) using high-performance liquid chromatography (HPLC, Waters 1525, Massachusetts), with a slight modification in the analytical method as previously reported. ${ }^{71}$ Briefly, analysis was performed using C18 column, $250 \mathrm{~mm} \times 4.6 \mathrm{~mm}$, particle size $5 \mu \mathrm{m}$ (Thermo Fisher Scientific, Massachusetts), via isocratic elution mode with a mobile phase of acetonitrile/water containing $0.1 \%$ trifluoroacetic acid at a ratio of 35:65 (\% v/v) with a flow rate of $1 \mathrm{~mL} / \mathrm{min}$ using a photodiode array detector at $310 \mathrm{~nm}$. Liquid-liquid extraction technique (ethyl acetate as extractive solvent) was adopted for the extraction of analytes from biological matrix. The calibration curve of PFD in plasma was plotted in the range of $100-3000 \mathrm{ng} / \mathrm{mL}$ with tinidazole $(1500$ $\mathrm{ng} / \mathrm{mL}$ ) as internal standard. The run time for each analysis was fixed at $30 \mathrm{~min}$ and data acquisition was performed using Breeze software.

4.11.2. Pharmacokinetic Analysis. A single-dose pharmacokinetic study was conducted on white albino rabbits with a PFD equivalent dose of $15 \mathrm{mg} / \mathrm{kg}$ body weight. The rabbits were classified into two groups $(n=3)$, group I (dosed with Pirfenex) and group II (dosed with F5), using oral gastric intubation tube. Blood samples $(1 \mathrm{~mL})$ were collected at predetermined time intervals from marginal ear vein at $0 \mathrm{~h}$ (predose) and at $0.5,1,1.5,2,3,4,6,9$, and $12 \mathrm{~h}$ (postdose) in K3-EDTA vials (Peerless Biotech Pvt. Ltd., Chennai, Tamil $\mathrm{Nadu}$, India). The blood samples were centrifuged for $10 \mathrm{~min}$, and at $-4{ }^{\circ} \mathrm{C}$, plasma was separated and analyzed using validated HPLC method as earlier described (Section 4.11.1). The pharmacokinetic parameters were calculated using zero moment noncompartment pharmacokinetic modeling. ${ }^{72}$ The procedure followed for animal housing and handling is provided in Section S.4.11.2, Supporting Information.

\section{ASSOCIATED CONTENT}

\section{Supporting Information}

The Supporting Information is available free of charge on the ACS Publications website at DOI: 10.1021/acsomega.8b00803.

Detailed discussion on the synthesis of carboxymethyl pullulan, its physicochemical characterization, experimental procedure for the preparation of microspheres, experimental procedure and mathematical models (OFW and KAS) for thermal kinetic study, experimental methods for in vitro enzymatic degradation study, procedure followed for cell culture and maintenance, protocol followed for conducting acute oral toxicity study, drug-release kinetics and mechanism modeling, stability study, animal housing and handling, in vitro enzymatic degradation result, acute oral toxicity result (hematological, serum biochemical parameters, and histopathological images), results of drug release kinetic modeling, results of stability study at accelerated conditions, proposed scheme for synthesis of CMP and its IPN microsphere, images of optical contact angle, $\mathrm{X}$-ray diffraction and differential scanning calorimeter spectra, weight loss thermogram (TG) and its derivative (DTA) spectra, graphical presentation of OFW and KAS model for CMP, PFD, placebo IPN microsphere (prepared using 1, 3, and $5 \mathrm{~mL} \mathrm{GA}$ ) and formulation (F5), contour plots from experimental design, histogram of formulation (F5), and dissolution profile of stability samples (PDF)

\section{AUTHOR INFORMATION}

\section{Corresponding Authors}

*E-mail: aghosh@bitmesra.ac.in, anim_1607@yahoo.co.in (A.G.).

*E-mail: biman.mandal@iitg.ac.in, mandal.biman@gmail.com (B.B.M.).

ORCID ${ }^{\circ}$

Biman B. Mandal: 0000-0003-3936-4621

Animesh Ghosh: 0000-0002-2990-4738

Notes

The authors declare no competing financial interest.

\section{ACKNOWLEDGMENTS}

A.G. sincerely acknowledges the financial support of SERB, New Delhi, through an EMR Individual Centric grant (File EMR/2014/000099). A.G. sincerely acknowledges support of ACS in form of ACS Author Reward (ARHD3C2R0F0D9K312QX9) which has been redeemed to purchase an open access license (Author Choice) for this article. The authors are grateful to Biophore India Pharmaceuticals Private Limited for the gift sample of PFD, and Gangwal Chemicals Private Limited, India, for supplying the gift sample of pullulan from the principle manufacturer Hayashibara Co. Ltd., Japan. B.B.M. greatly acknowledges funding support from Department of Biotechnology (DBT) and Department of Science and Technology (DST), Govt. of India. The authors acknowledge Biomaterial and Tissue Engineering Laboratory, Department of Biosciences and Bioengineering, IIT Guwahati, for providing necessary facilities for conducting cytocompatibility studies and Department of Pharmaceutical Sciences and Technology along with CIF 
facility of BIT Mesra, Ranchi, for providing all other required facilities.

\section{ABBREVIATIONS}

PVA, poly(vinyl alcohol); GA, glutaraldehyde; PFD, pirfenidone; CMP, carboxymethyl pullulan; IPN, interpenetrating polymeric network; IPF, idiopathic pulmonary fibrosis; MTT, 3-(4,5-dimethylthiazol-2-yl)-2,5-diphenyltetrazolium bromide

\section{REFERENCES}

(1) Dubey, R. D.; Saneja, A.; Gupta, P. K.; Gupta, P. N. Recent advances in drug delivery strategies for improved therapeutic efficacy of gemcitabine. Eur. J. Pharm. Sci. 2016, 93, 147-162.

(2) Future Market Insights (FMI) Report, 2017. https://www. prnewswire.com/news-releases/solid-dosage-forms-will-remainpreferred-in-the-global-oral-controlled-release-drug-deliverytechnology-market-637852753.html (last assessed May 02, 2018).

(3) Santos, L. F.; Correia, I. J.; Silva, A. S.; Mano, J. F. Biomaterials for drug delivery patches. Eur. J. Pharm. Sci. 2018, 118, 49-66.

(4) Degen, L.; Phillips, S. Variability of gastrointestinal transit in healthy women and men. Gut 1996, 39, 299-305.

(5) Gupta, U.; Perumal, O. Dendrimers and Its Biomedical Applications. In Natural and Synthetic Biomedical Polymers; Elsevier, 2014; pp 243-257.

(6) Ahsan, S. M.; Thomas, M.; Reddy, K. K.; Sooraparaju, S. G.; Asthana, A.; Bhatnagar, I. Chitosan as biomaterial in drug delivery and tissue engineering. Int. J. Biol. Macromol. 2018, 110, 97-109.

(7) Kumar, A.; Rao, K. M.; Han, S. S. Application of xanthan gum as polysaccharide in tissue engineering: A review. Carbohydr. Polym. 2018, 180, 128-144.

(8) Ali, A.; Ahmed, S. A Review on Chitosan and its Nanocomposites in Drug Delivery. Int. J. Biol. Macromol. 2018, 109, 273286.

(9) Vijan, V.; Kaity, S.; Biswas, S.; Isaac, J.; Ghosh, A. Microwave assisted synthesis and characterization of acrylamide grafted gellan, application in drug delivery. Carbohydr. Polym. 2012, 90, 496-506.

(10) Kaity, S.; Isaac, J.; Kumar, P. M.; Bose, A.; Wong, T. W.; Ghosh, A. Microwave assisted synthesis of acrylamide grafted locust bean gum and its application in drug delivery. Carbohydr. Polym. 2013, 98, 1083-1094.

(11) Prabaharan, M. Prospective of guar gum and its derivatives as controlled drug delivery systems. Int. J. Biol. Macromol. 2011, 49, 117-124.

(12) Al-Karawi, A. J. M.; Al-Daraji, A. H. R. Preparation and using of acrylamide grafted starch as polymer drug carrier. Carbohydr. Polym. 2010, 79, 769-774.

(13) Shao, Y.; Li, L.; Gu, X.; Wang, L.; Mao, S. Evaluation of chitosan-anionic polymers based tablets for extended-release of highly water-soluble drugs. Asian J. Pharm. Sci. 2015, 10, 24-30.

(14) Viridén, A.; Larsson, A.; Schagerlöf, H.; Wittgren, B. Model drug release from matrix tablets composed of HPMC with different substituent heterogeneity. Int. J. Pharm. 2010, 401, 60-67.

(15) Mittermayr, R.; Slezak, P.; Haffner, N.; Smolen, D.; Hartinger, J.; Hofmann, A.; Schense, J.; Spazierer, D.; Gampfer, J.; Goppelt, A.; Redl, H. Controlled release of fibrin matrix-conjugated platelet derived growth factor improves ischemic tissue regeneration by functional angiogenesis. Acta Biomater. 2016, 29, 11-20.

(16) Gander, B.; Gurny, R.; Doelker, E.; Peppas, N. A. Effect of polymeric network structure on drug release from cross-linked poly(vinyl alcohol) micromatrices. Pharm. Res. 1989, 6, 578-584.

(17) Martinez, A. W.; Caves, J. M.; Ravi, S.; Li, W.; Chaikof, E. L. Effects of crosslinking on the mechanical properties, drug release and cytocompatibility of protein polymers. Acta Biomater. 2014, 10, 2633.

(18) Carbinatto, F. M.; de Castro, A. D.; Evangelista, R. C.; Cury, B. $S$. Insights into the swelling process and drug release mechanisms from cross-linked pectin/high amylose starch matrices. Asian J. Pharm. Sci. 2014, 9, 27-34.
(19) Rokhade, A. P.; Agnihotri, S. A.; Patil, S. A.; Mallikarjuna, N. N.; Kulkarni, P. V.; Aminabhavi, T. M. Semi-interpenetrating polymer network microspheres of gelatin and sodium carboxymethyl cellulose for controlled release of ketorolac tromethamine. Carbohydr. Polym. 2006, 65, 243-252.

(20) Matricardi, P.; Di Meo, C.; Coviello, T.; Hennink, W. E.; Alhaique, F. Interpenetrating polymer networks polysaccharide hydrogels for drug delivery and tissue engineering. Adv. Drug Delivery Rev. 2013, 65, 1172-1187.

(21) Soni, S. R.; Ghosh, A. Exploring pullulan-poly(vinyl alcohol) interpenetrating network microspheres as controlled release drug delivery device. Carbohydr. Polym. 2017, 174, 812-822.

(22) Shaw, J.; Marshall, T.; Morris, H.; Hayton, C.; Chaudhuri, N. Idiopathic pulmonary fibrosis: a holistic approach to disease management in the antifibrotic age. J. Thorac. Dis. 2017, 9, 4700.

(23) Pirfenidone. USFDA Reviews. Pharmacology Review(s); Center for Drug Evaluation and Research, 2014; pp 1-496.

(24) Jana, S.; Sharma, R.; Maiti, S.; Sen, K. K. Interpenetrating hydrogels of O-carboxymethyl Tamarind gum and alginate for monitoring delivery of acyclovir. Int. J. Biol. Macromol. 2016, 92, 1034-1039.

(25) Kaity, S.; Ghosh, A. Carboxymethylation of locust bean gum: application in interpenetrating polymer network microspheres for controlled drug delivery. Ind. Eng. Chem. Res. 2013, 52, 1003310045.

(26) Pushpamalar, V.; Langford, S.; Ahmad, M.; Lim, Y. Optimization of reaction conditions for preparing carboxymethyl cellulose from sago waste. Carbohydr. Polym. 2006, 64, 312-318.

(27) Sharma, B. R.; Kumar, V.; Soni, P. Carbamoylethylation of Cassia tora gum. Carbohydr. Polym. 2003, 54, 143-147.

(28) Aminabhavi, T. M.; Nadagouda, M. N.; More, U. A.; Joshi, S. D.; Kulkarni, V. H.; Noolvi, M. N.; Kulkarni, P. V. Controlled release of therapeutics using interpenetrating polymeric networks. Expert Opin. Drug Delivery 2015, 12, 669-688.

(29) Whistler, R. L.; BeMiller, J. Alkaline Degradation of Polysaccharides. Advances in Carbohydrate Chemistry; Elsevier, 1958; pp 289-329.

(30) Alekhina, M.; Mikkonen, K. S.; Alén, R.; Tenkanen, M.; Sixta, $\mathrm{H}$. Carboxymethylation of alkali extracted xylan for preparation of bio-based packaging films. Carbohydr. Polym. 2014, 100, 89-96.

(31) Yuen, S.-N.; Choi, S.-M.; Phillips, D. L.; Ma, C.-Y. Raman and FTIR spectroscopic study of carboxymethylated non-starch polysaccharides. Food Chem. 2009, 114, 1091-1098.

(32) Mansur, H. S.; Oréfice, R. L.; Mansur, A. A. Characterization of poly(vinyl alcohol)/poly(ethylene glycol) hydrogels and PVA-derived hybrids by small-angle X-ray scattering and FTIR spectroscopy. Polymer 2004, 45, 7193-7202.

(33) Badr, Y. A.; El-Kader, K. M. A.; Khafagy, R. M. Raman spectroscopic study of CdS, PVA composite films. J. Appl. Polym. Sci. 2004, 92, 1984-1992.

(34) Masuda, K.; Kaji, H.; Horii, F. CP/MAS ${ }^{13} \mathrm{C}$ NMR analyses of hydrogen bonding and the chain conformation in the crystalline and noncrystalline regions for poly(vinyl alcohol) films. J. Polym. Sci., Part B: Polym. Phys. 2000, 38, 1-9.

(35) Yang, H.; Hu, S.; Horii, F.; Endo, R.; Hayashi, T. CP/MAS ${ }^{13} \mathrm{C}$ NMR analysis of the structure and hydrogen bonding of meltcrystallized poly(vinyl alcohol) films. Polymer 2006, 47, 1995-2000.

(36) Kajjari, P. B.; Manjeshwar, L. S.; Aminabhavi, T. M. Semiinterpenetrating polymer network hydrogel blend microspheres of gelatin and hydroxyethyl cellulose for controlled release of theophylline. Ind. Eng. Chem. Res. 2011, 50, 7833-7840.

(37) Kajjari, P. B.; Manjeshwar, L. S.; Aminabhavi, T. M. Novel interpenetrating polymer network hydrogel microspheres of chitosan and poly(acrylamide)-grafted-guar gum for controlled release of ciprofloxacin. Ind. Eng. Chem. Res. 2011, 50, 13280-13287.

(38) Park, J. W.; Oh, S. C.; Lee, H. P.; Kim, H. T.; Yoo, K. O. A kinetic analysis of thermal degradation of polymers using a dynamic method. Polym. Degrad. Stab. 2000, 67, 535-540. 
(39) Jakab, E.; Faix, O.; Till, F.; Székely, T. The effect of cations on the thermal decomposition of lignins. J. Anal. Appl. Pyrolysis 1993, 25, 185-194.

(40) Gómez, I.; Otazo, E. M.; Hernández, H.; Rubio, E.; Varela, J.; Ramírez, M.; Barajas, I.; Gordillo, A. J. Thermal degradation study of PVA derivative with pendant phenylthionecarbamate groups by DSC/ TGA and GC/MS. Polym. Degrad. Stab. 2015, 112, 132-136.

(41) Chrissafis, K.; Paraskevopoulos, K.; Bikiaris, D. Thermal degradation kinetics of the biodegradable aliphatic polyester, poly(propylene succinate). Polym. Degrad. Stab. 2006, 91, 60-68.

(42) Wu, S.-J.; Chen, J. Preparation of maltotriose from fermentation broth by hydrolysis of pullulan using pullulanase. Carbohydr. Polym. 2014, 107, 94-97.

(43) Edwards, C. H.; Warren, F. J.; Milligan, P. J.; Butterworth, P. J.; Ellis, P. R. A novel method for classifying starch digestion by modelling the amylolysis of plant foods using first-order enzyme kinetic principles. Food Funct. 2014, 5, 2751-2758.

(44) Butterworth, P. J.; Warren, F. J.; Grassby, T.; Patel, H.; Ellis, P. $\mathrm{R}$. Analysis of starch amylolysis using plots for first-order kinetics. Carbohydr. Polym. 2012, 87, 2189-2197.

(45) Reddy, K. M.; Babu, V. R.; Sairam, M.; Subha, M. C. S.; Mallikarjuna, N. N.; Kulkarni, P. V.; Aminabhavi, T. M. Development of chitosan-guar gum semi-interpenetrating polymer network microspheres for controlled release of cefadroxil. Des. Monomers Polym. 2006, 9, 491-501.

(46) Das, D.; Das, R.; Ghosh, P.; Dhara, S.; Panda, A. B.; Pal, S. Dextrin cross linked with poly(HEMA): a novel hydrogel for colon specific delivery of ornidazole. RSC Adv. 2013, 3, 25340-25350.

(47) Angadi, S. C.; Manjeshwar, L. S.; Aminabhavi, T. M. Coated interpenetrating blend microparticles of chitosan and guar gum for controlled release of isoniazid. Ind. Eng. Chem. Res. 2013, 52, 63996409.

(48) Babu, V. R.; Sairam, M.; Hosamani, K.; Aminabhavi, T. Preparation and characterization of novel semi-interpenetrating 2hydroxyethyl methacrylate-g-chitosan copolymeric microspheres for sustained release of indomethacin. J. Appl. Polym. Sci. 2007, 106, $3778-3785$.

(49) Rokhade, A. P.; Kulkarni, P. V.; Mallikarjuna, N. N.; Aminabhavi, T. M. Preparation and characterization of novel semiinterpenetrating polymer network hydrogel microspheres of chitosan and hydroxypropyl cellulose for controlled release of chlorothiazide. J. Microencapsulation 2009, 26, 27-36.

(50) Reddy, K. M.; Babu, V. R.; Rao, K. S. V. K.; Subha, M. C. S.; Rao, K. C.; Sairam, M.; Aminabhavi, T. M. Temperature sensitive semi-IPN microspheres from sodium alginate and $\mathrm{N}$-isopropylacrylamide for controlled release of 5-fluorouracil. J. Appl. Polym. Sci. 2008, 107, 2820-2829.

(51) Varelas, C. G.; Dixon, D. G.; Steiner, C. A. Zero-order release from biphasic polymer hydrogels. J. Controlled Release 1995, 34, 185192.

(52) Wagner, J. G. Interpretation of percent dissolved-time plots derived from in vitro testing of conventional tablets and capsules. J. Pharm. Sci. 1969, 58, 1253-1257.

(53) Costa, P.; Lobo, J. M. S. Modeling and comparison of dissolution profiles. Eur. J. Pharm. Sci. 2001, 13, 123-133.

(54) Mulye, N.; Turco, S. A simple model based on first order kinetics to explain release of highly water soluble drugs from porous dicalcium phosphate dihydrate matrices. Drug Dev. Ind. Pharm. 1995, 21, 943-953.

(55) Hixson, A.; Crowell, J. Dependence of reaction velocity upon surface and agitation. Ind. Eng. Chem. 1931, 23, 1160-1168.

(56) Higuchi, T. Mechanism of sustained-action medication. Theoretical analysis of rate of release of solid drugs dispersed in solid matrices. J. Pharm. Sci. 1963, 52, 1145-1149.

(57) Korsmeyer, R. W.; Gurny, R.; Doelker, E.; Buri, P.; Peppas, N. A. Mechanisms of solute release from porous hydrophilic polymers. Int. J. Pharm. 1983, 15, 25-35.
(58) Eyler, R.; Klug, E.; Diephuis, F. Determination of degree of substitution of sodium carboxymethylcellulose. Anal. Chem. 1947, 19, 24-27.

(59) Kaity, S.; Ghosh, A. Facile preparation of acrylamide grafted locust bean gum-poly(vinyl alcohol) interpenetrating polymer network microspheres for controlled oral drug delivery. J. Drug Delivery Sci. Technol. 2016, 33, 1-12.

(60) Flynn, J. H.; Wall, L. A. General treatment of the thermogravimetry of polymers. J. Res. Natl. Bur. Stand., Sect. A 1966, 70A, 487-523.

(61) Ozawa, T. A new method of analyzing thermogravimetric data. Bull. Chem. Soc. Jpn. 1965, 38, 1881-1886.

(62) Akahira, T.; Sunose, T. Method of Determining Activation Deterioration Constant of Electrical Insulating; Research Report; Institute of Technology, 1971; Vol. 16, pp 22-31.

(63) Kissinger, H. E. Reaction kinetics in differential thermal analysis. Anal. Chem. 1957, 29, 1702-1706.

(64) Ali, G.; Rihouey, C.; Le Cerf, D.; Picton, L. Effect of carboxymethyl groups on degradation of modified pullulan by pullulanase from Klebsiella pneumoniae. Carbohydr. Polym. 2013, 93, 109-115.

(65) Saqib, A. A. N.; Whitney, P. J. Differential behaviour of the dinitrosalicylic acid (DNS) reagent towards mono-and di-saccharide sugars. Biomass Bioenergy 2011, 35, 4748-4750.

(66) Kumar, J. P.; Mandal, B. B. Antioxidant potential of mulberry and non-mulberry silk sericin and its implications in biomedicine. Free Radic. Biol. Med. 2017, 108, 803-818.

(67) Joseph, C. M.; Reardon, P. J. T.; Konwarh, R.; Knowles, J. C.; Mandal, B. B. Mimicking Hierarchical Complexity of the Osteochondral Interface Using Electrospun Silk-Bioactive Glass Composites. ACS Appl. Mater. Interfaces 2017, 9, 8000-8013.

(68) Kumar, P. M.; Ghosh, A. Development and evaluation of silver sulfadiazine loaded microsponge based gel for partial thickness (second degree) burn wounds. Eur. J. Pharm. Sci. 2017, 96, 243-254.

(69) Kaity, S.; Isaac, J.; Ghosh, A. Interpenetrating polymer network of locust bean gum-poly(vinyl alcohol) for controlled release drug delivery. Carbohydr. Polym. 2013, 94, 456-467.

(70) Isaac, J.; Kaity, S.; Ganguly, S.; Ghosh, A. Microwave-induced solid dispersion technology to improve bioavailability of glipizide. $J$. Pharm. Pharmacol. 2013, 65, 219-229.

(71) Shi, S.; Wu, J.; Chen, H.; Chen, H.; Wu, J.; Zeng, F. Single-and Multiple-Dose Pharmacokinetics of Pirfenidone, an Antifibrotic Agent, in Healthy Chinese Volunteers. J. Clin. Pharmacol. 2007, 47, $1268-1276$

(72) Kaity, S.; Ghosh, A. Comparative bio-safety and in vivo evaluation of native or modified locust bean gum-PVA IPN microspheres. Int. J. Biol. Macromol. 2015, 72, 883-893. 